\title{
Wild Edible Plants of Nutritional and Medicinal Significance to The Tribes of Palghar, Maharashtra, India
}

\author{
Yatish Lele, Bhargavi Thorve, Swati Tomar, Anjali Parasnis
}

Yatish Lele is M.Sc in Environmental Science. Working in subjects related to forestry and biodiversity as Associate Fellow in Forestry and Biodiversity Division, The Energy and Resources Institute, Delhi, India, Email : Yatish.Lele@teri.res.in (Author for Correspondence) ORCID: https:/ / orcid.org/0000-0002-1800-2835

Bhargavi Thorve is M.Sc Environmental Science. Working in the Food and Nutrition Security Division, The Energy and Resources Institute, Maharashtra, India

Swati Tomar is M.Sc Environmental Science. Working in the Food and Nutrition Security Division, The Energy and Resources Institute, Maharashtra, India

Anjali Parasnis is Associate Director and Senior Fellow at Food and Nutrition Security Division, The Energy and Resources Institute, Maharashtra, India

\begin{abstract}
Wild edible plants (WEP) are an important component from the perspective of tribal diet. There are various traditional practices and beliefs in relation to the use of wild edibles among various tribal communities in Maharashtra. In this study, the WEP found in Jawhar block of Palghar district and detailed information on the local usage for medicine or food purpose has been documented. With this, the traditional methods of preparation, collection and storage of these edible wild plants has also been documented. The present paper presents a total of 162 species of WEP, out of which almost $74 \%$ are consumed as food, $14 \%$ possess medicinal significance while $12 \%$ of the species exhibit both dietary and medicinal significance. This type of study could contribute in recording the traditional heritage of food culture and generate awareness about the importance of wild edible species. Documentation of these wild plant species can help in commercialization and domestication of the wild varieties and their entry into urban marketplaces to generate higher revenue for the farmers. Wild edibles could prove to be a remedy to food scarcity, a source of nutritional security and improve the economy in tribal areas.
\end{abstract}

Keywords : Tribal communities, Wild edible plants, Traditional knowledge, Jawhar, Western Ghats

\section{Introduction}

Over the millennia, forests have played a key role as a source of food, fodder, fuel and medicine in the lives and livelihood of the forest dwelling communities. Although the societies primarily depend on agriculture for food, millions of people like the forest dwelling communities and rural population, have traditionally depended on the wild plant resources (Panda, 2014). Wild edible plants (WEP) are widely used for various purposes mostly as a source of food and medicine by the tribal communities all across the world. These tribal communities have acquired immense knowledge about the use of wild plant species based on the local needs, preferences, customs, and habit (Jain and
Tiwari, 2012). Even today, indigenous people continue to follow many traditions and have long experience and close association with the nature. For example, Vietnamese commonly use Chinese herbal medicine and a set of indigenous folk practices referred to as "Southern medicine" in an effort to cure any disease (Jenkins, 1996) whereas, 'Ayurveda' in Indian Folkmedicine is still an important method of treatment using the plant resources (Deshmukh and Waghmode, 2011).

A number of wild plants significantly contribute to the human diet and food security across the world. In central Italy, 126 wild plants from 39 families are consumed (JmanRedzic, 2006) and wild greens are eaten raw in salads, or in boiled mixtures, as 'blood 
cleansing' and 'intestine cleansing' agents (Pieroni, 2000). In Vietnam, over 90 species of WEP have been documented (Ogle et.al, 2003). Several studies on dietary uses of edible wild plants are forthcoming to tackle malnutrition and famine conditions faced by the tribal population of Nigeria which is prone to droughts and crop failure (Lockett et al, 2000). Hence the WEP are also significant and usually considered as an Emergency food during the adverse conditions such as floods, droughts and famine.

Similarly, it has also been estimated that there are around 800 varieties of wild species being consumed in India (Nivedita, 2017). The Indian Himalaya reveals the richest biodiversity of WEP with over 675 wild plant species used as food and their various parts are either consumed raw, roasted, boiled, fried, cooked or in the form of oil, spice, seasoning material, jams, and pickles by the native communities (Samant, 1997). The Garo tribe in Meghalaya uses around 38 species of wild plants as vegetables and 33 species are consumed raw or cooked (Singh et al, 2012); 28 species of wild plants are consumed as vegetables by aboriginal of Vindhyan plateau in Central India and over 45 plant species are consumed as emergency food by the tribes inhibiting Pachmarhi biosphere reserve in Madhya Pradesh (Jain and Tiwari, 2012). On the other hand, an ethnobotanical study on edible wild plants in a few chosen districts of Maharashtra reports 29 wild edible fruit plants consumed by tribal communities from Kalsubai- Harishchandragad wildlife sanctuary (Deshmukh and Vidya, 2010); 101 species were recorded used by tribes residing in Toranmal plateau of Maharashtra (Sharma and Mujumdar 2003) and 30 plant species, used by the tribal and non-tribal of Nasik district for ethnomedicinal purposes was recorded by Patil and Patil (2005).

This clearly indicates that the wild edibles form a major and prominent source of food and medicine in the daily routine of the tribal and non-tribal. Wild edibles such as tubers and corms are also frequently consumed to fulfil the critical gaps created by the agricultural shortfall, natural disasters, disease epidemics and famine and thus act a response to emergency (Belcher, 2005). However owing to the land use changes and yearlong availability of fruits and vegetables in the local markets, the use of wild edibles has been restricted which shall in turn threaten the traditional knowledge of the use of these wild plants. As a result, the ethnobotanical studies need to be carried out to document the important sources of nutrition which played an important role in their diet and medicine.

This study documents the traditional knowledge of wild edibles in the Jawhar region of Maharashtra, India and provides an inventory of wild plants consumed by the tribal communities of Jawhar block of Palghar district in Maharashtra. The plants have been recorded along with their availability, seasonality, uses and traditional recipes.

\section{Methodology}

\section{Study Area}

Jawhar block from Palghar district, Maharashtra state, India was identified as the site for conducting the study. Known as "Mahabaleshwar (A famous hill station in India) of Palghar district", Jawhar taluka is located at $19.92^{\circ} \mathrm{N} 73.23^{\circ} \mathrm{E}$ at the height of approx. 473 meters above sea level (Fig. 1). The area receives an average rainfall of around $3000 \mathrm{~mm}$ (Chotte et al, 2014). Being part of the Northern Western Ghats, the area is rich in biodiversity with a moist deciduous type of forest ecosystem (Champion and Seth, 1968). The region is inhabited by different tribes like Thakur, Mahadev Koli, Warli, Katkaris and Kokana who are partly dependent on different plants species for food and medicinal purposes.

Palghar district is well known for the WEP consumed by the tribal population (Sonawane et al., 2012; Chotte et al, 2014; Oak et al 2015; Shivprasad et al2016). But there is no proper documentation and validation of the WEP of Jawhar region. The tribal communities in Jawhar also depend on the WEP for their dietary and medicinal purposes apart from the agricultural produce. These WEP are largely harvested

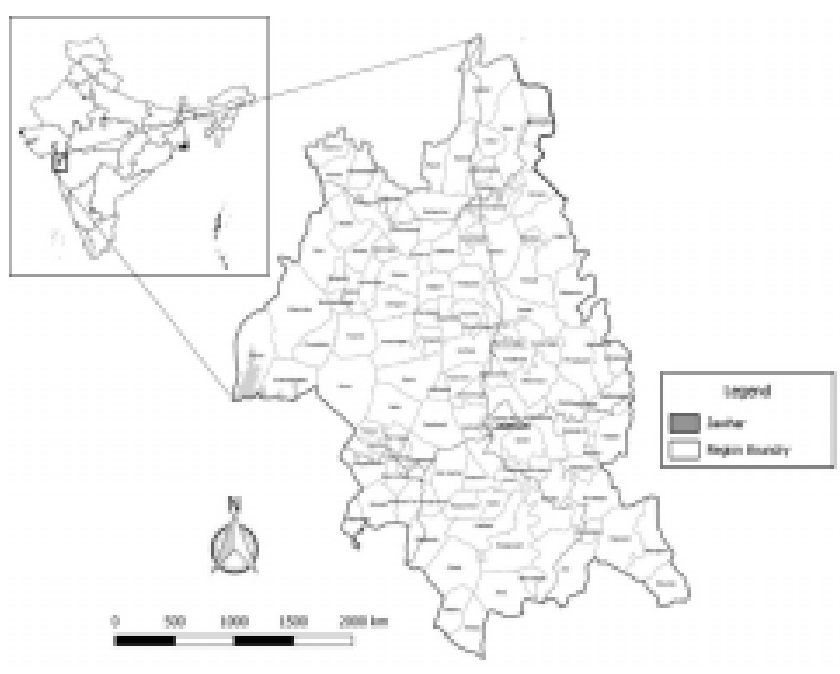

Map 1: Location of the study site 
from the local forest areas present around villages. Today, expansion of agricultural area and over-exploitation of the Non Timber Forest Produce (NTFP) have resulted in the degradation and deforestation which may threaten the ecological and biological value of the remaining tracks of forests in future (Belcher, 2005).

One of the major issues faced by the tribal population in this region is malnutrition. Malnourishment remains a major problem in the region mostly in the women and children below age group of 6 years. Studies have reported the issue of malnourishment to be severe in case of infants and children (Hatekar and Rode., 2003; Dimri et.al, 2012). One of the possible reasons for this severity could be lack of knowledge about healthy diet, ignorance towards wellbeing and proper sanitation or lack of food variety and absence of purchase power to procure nutritious food. Today, local livelihoods are mainly based on the small scale farming with cultivating sustenance crops such as rice, nachni etc; fruit crops such as banana and chickoo. The other sources of income mostly include labour wages in the nearby cities such as Thane and Mumbai.

\section{Methodology}

The study was conducted from December 2014 to March 2016. Extensive review of literature was undertaken in order to collate information about the wild edible species from Jawhar block. Focus Group Discussions (FGD) were conducted in 25 tribal villages namely Tasupada, Madvihira, Kahandolpada, Umbrachapada, Bhagada, Savarpada, Malghar, Khuripada, Chibhadepada, Kogade, Jamsar, Sarsun, Umbervihir, Khambala, Behedpada, Dhodipada, Barwadpada, Pachgud, Kalamvihira and Kasatwadi which were selected by stratified random sampling. The local names of the plant species and their significance to the community was documented using a questionnaire. The discussions consisted of men, women, youth, elderly people, local medicine men (locally known as Vaidu) as well as plant experts (botanists) to understand the varied significance of wild edibles to each group (in this case total, $\mathrm{N}=323$ ).

Participatory transect surveys were undertaken in the forest areas with the help of the local tribes to identify and validate the plant species. Flora of Maharashtra aided in the identification of plants on field (Almeida, 1996, 1998, 2001a,b; 2003a, b; 2009 a, b). The surveys were undertaken seasonally as the floral pattern is subjected to change as per the season. The points to be surveyed in the forest were marked using Google Earth software. On field, these points were identified, tracked and marked using a Global Posi- tioning System (GPS). The relevant plants observed at random were also recorded to collate and update the checklist. This approach helped document all the wild edibles present in the area irrespective of their significance to the tribal communities in the vicinity.

\section{Results and Discussion}

\section{WEP and Perceptions}

WEP are being consumed by all the strata of the tribal community throughout the year. A total of 162 species of WEP were reported from the survey. The details of the reported plant species such as the local names of the plant, season of availability and significance of the wild edible plant and the method of consumption is provided below in Table 1 .

The study indicated plants belonging to 65 families out of which maximum plants consumed were from Leguminosae family (19 species). The top ten families following Leguminosae were Cucurbitaceae (12 species), Malvaceae (10 species), Dioscoreaceae (7 species), Amaranthaceae (6 species), Araceae (6 species), Lamiaceae (6 species), Poaceae (5 species), Rubiaceae (5 species) and Apocynaceae (4 species). As it could be observed from Fig. 2, trees have constituted 34\% of the WEP species followed by herbs (29\%), climbers $(23 \%)$, shrubs (12\%), Fungus(mushrooms) (1\%) and fern species $(1 \%)$.

Although the WEP form a crucial part of the diet of the tribal communities, very few of them were aware about of the nutritional aspect of the wild edibles. The knowledge of medicinal uses of plants species was restricted to the older generation or the Vaidu'. It was found that out of the 162 species of wild edibles, almost $74 \%$ of the wild edibles were consumed as food and $14 \%$ of the species possess medicinal significance

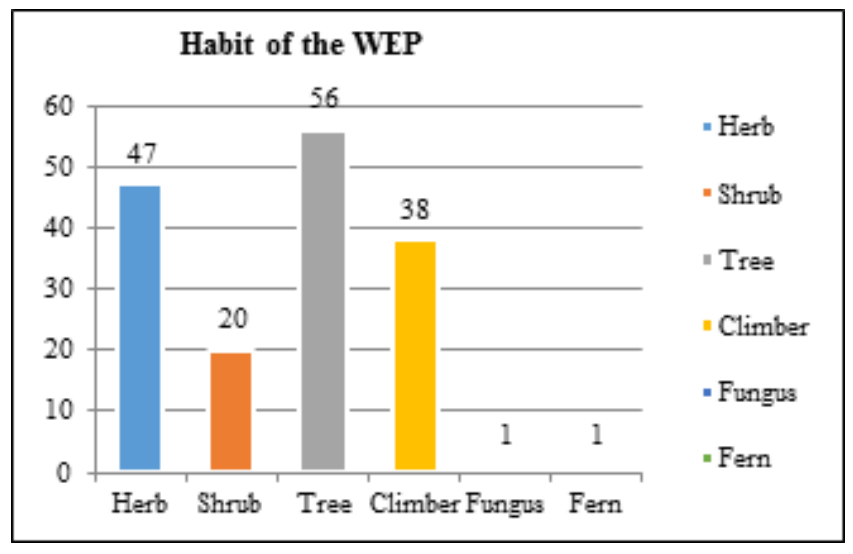

Graph 1 : Life forms of the WEP recorded in Jawhar 
while $12 \%$ of the species are characterised for both dietary and medicinal significance.

\section{Seasonal availability of wild food resources}

Seasons play an important role in the pattern of consumption of wild edibles by the tribal communities. A total of $48 \%$ species are seen in monsoon (JuneSeptember) while $20 \%$ plants are found throughout the year, $18 \%$ in summers (February- May) and $14 \%$ in winters (October- January) (Fig. 3).

Different processes of the plant such as flowering, fruiting, and leafing seasonal and hence different plant parts are consumed in different seasons. Of these $30 \%$ consumption was of fruits, followed by leaves $(27 \%)$, seeds $(12 \%)$, flowers $(8 \%)$, tubers $(7 \%)$ and followed by others (Fig.4).

The analysis shows that most of the wild edibles are consumed during the monsoon. Most of the fruits, flowers and leaves of different plant species are harvested for consumption during monsoons. The food items being highly perishable are mostly consumed immediately after harvesting while some species are stored in the form of pickles or dried forms (Chotte A, 2014). Tree species such as Pterocarpus marsupium, Anogeissus latifolia are perennial thus the leaves, bark and sap of these plants are utilized on a regular basis. The months of winter and summer are most vulnerable to food shortages due to water scarcity and less availability of edible plant species in the wild. In such situations, tubers are the preferred due to their availability in the dry season. The tribal communities depend on the agricultural produce during this time of the year.

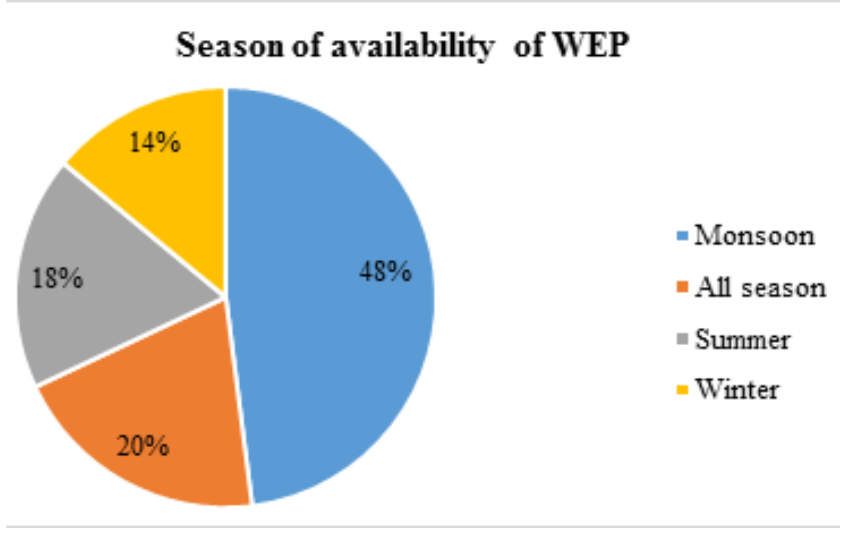

Graph 2: Season of availability of the WEP for consumption

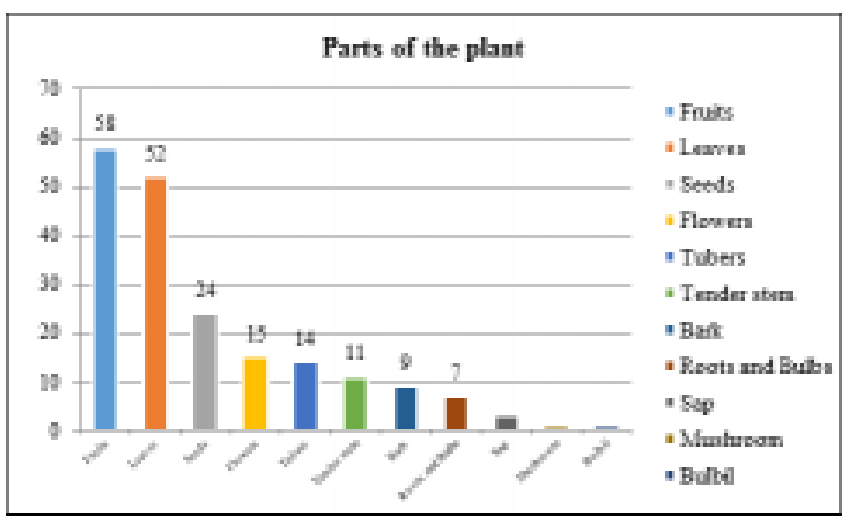

Graph 3: Parts of the plant consumed by the tribal communities of Jawhar

\section{Consumption of Significant Species}

The tribes in Kasatwadi village consume a fern species, Athyrium hohenackerianum, (Akri) which is a unique observation from the study area. The studies reporting the consumption of WEP from Maharashtra have not yet reported such observation from different parts of the state. Thus this is the first of its kind recorded observation of consumption of fern as food. But this observation was recorded only from one village, Kasatwadi, while communities from other villages are unaware about the significance of the fern. Another species, Justicia procumbens (Sharambal) was also found to be consumed only in the Kasatwadi village. The reason for the same was unknown. Species consumed by different villages also varied. Villages situated near river were found to consume aquatic plant species such as Cryptocoryne spiralis (Sol) and Marsilea quadrifolia (Girjala) whereas villages situated up-hill did not have access to these and thus were ignorant about its availability.

Some of the plant species have also been domesticated such as Momordica dioica (Karli/Karele), Dioscorea bulbifera (Kadukand), Amorphophallus paeoniifolius (Suran), Curcuma longa (Halad/Haldi) and Basella alba (Mayalu/ Indian Spinach) which are cultivated in the backyard / kitchen gardens. Germplasm of these plants are stored until the next season of plantation. Few wild edibles are found to be consumed only in few villages due to the geographical differences, lack of availability and accessibility and lack of knowledge about the plant species. It was also found that few wild edibles such as tubers, bamboo, leafy vegetable, fruits and flowers also contribute to the economics of the tribal communities. The tribes sell the WEP in the local market at Jawhar or sell it directly by 
setting up small stalls at the road sides or highways.

\section{Conclusion}

The regular diet of the tribal people of Jawhar consist of rice, finger millet (nachni/raagi), urad and few locally available vegetables. They mostly depend on rain-fed agriculture for food and sell the surplus. Forest land is gradually getting converted into agriculture land, with the growing demand for food due to increasing population, but climatic variations are negatively impacting the agricultural produce. Shifting cultivation is the common practice and increasing deforestation and mono-culture are changing the landscape which is a threat to the availability and abundance of wild edibles. This could also be one of the reasons why the tribal communities are increasingly dependent for food on agricultural produce available in the market. Moreover, lack of knowledge about WEP in the younger generation and the lack of livelihood opportunities leads to migration which again results in to lesser consumption of wild edibles.

Traditionally used wild edibles have enormous medicinal potential and could be an excellent source of nutrition and vitamins as a supplementary food. This immense knowledge gained through trial and error practices over several decades needs to be documented as they are an integral part of the Indian culture. It has been reported that out of an estimated portfolio of 30,000 edible plant species recorded worldwide, man depends only on 12 crops for the bulk of its nutritional requirements (Padulosi et al, 2011). This implies the great potential of wild edibles in the context of food security. The decreased use of wild edibles due to modernization and lack of knowledge among the younger generation are the major challenges. Thus it is important to conduct various studies on the commercial potential for ensuring the conservation and sustainable utilization of these species to hold a food-secure future.

\section{References}

Almeida, M. R. (1996) Flora of Maharashtra (Ranunculaceae to Connaraceae). Volume 1.St. Xavier's College, Mumbai, 294 pp.

Almeida, M. R. (1998) Flora of Maharashtra (Fabaceae to Apiaceae). Volume 2. St.Xavier's College, Mumbai, 372 pp.

Almeida, M. R. (2001a) Flora of Maharashtra (Cuscutaceae to Martyniaceae). Volume 3b.St. Xavier's College, Mumbai, 301-464 pp.

Almeida, M. R. (2001b) Flora of Maharashtra (Rubiaceae to Ehretiaceae). Volume 3a. St.Xavier's
College, Mumbai, 300 pp.

Almeida, M. R. (2003a) Flora of Maharashtra (Bischofiaceae to Ceratophyllaceae).Volume 4b. St. Xavier's College, Mumbai, 279399 pp.

Almeida, M. R. (2003b) Flora of Maharashtra (Acanthaceae to Balanophoraceae). Volume4a. St. Xavier's College, Mumbai, 278 pp.

Almeida, M. R. (2009a) Flora of Maharashtra (Hydrocharitaceae to Typhaceae). Volume5a. St. Xavier's College, Mumbai, 245 pp.

Almeida, M. R. (2009b) Flora of Maharashtra (Araceae to Cyperaceae). Volume 5b. St.Xavier's College, Mumbai, 246495 pp

Almeida, M. R. (2003). Flora of Maharashtra Vol. IV. Mumbai: Orient Press..

Belcher, B., Ruiz Perez, M. and Achdiawan, R. (2005). Global patterns and trends in the use and management of commercial NTFPs: implications for livelihoods and conservation, World development, 33(9), 1435-1452.

Champion, H. G. and Seth, S. K. (1968). A Revised Survey of Forest Types of India, Govt. of India Press, New Delhi, p. 404.

Chotte, A., Patil, S., and Kulkarni, D. K. (2014). Unconventional wild fruits and processing in tribal area of Jawhar, Thane District, Bioscience Discovery, 5 (1), 19-23.

Deshmukh, B. and Waghmode, A. (2011). Role of wild edible fruits as a food resource: Traditional knowledge, International Journal of Pharmacy and Life Sciences, 2(7) 919- 924.

Deshmukh, B. S. and Vidya, S. (2010). Fruits in the wilderness: a potential of the local food resource, International Journal of Pharma and Bio Sciences, 1(2). 1- 5.

Dimri, A., Pohekar, A. and Thakur. S.N. Scoping malnutrition in selected talukas of Thane district for possible intervention through nutrition supplements,. Centre for Technology Alternatives for Rural Areas. IIT Bombay.

Hatekar, N. and Rode, S. (2003). Quietly They Die: A Study of Malnourishment Related Deaths in Mumbai City, Working Paper 1/2003. Department of Economics, University of Mumbai

Jain A. K and Tiwari P (2012). Nutritional value of some traditional edible plants used by tribal communities during emergency with reference to Central India, Indian Journal of Traditional Knowledge, 5157.

Jenkins, C.L. (1996). Health care access and preventive care among Vietnamese immigrants: do traditional beliefs and practices pose barriers?,Social science 
and medicine, 43(7) 1049-1056.

Jman Redzic, S. (2006). Wild edible plants and their traditional use in the human nutrition in BosniaHerzegovina, Ecology of Food and Nutrition, 45(3). 189-232.

Lockett, T, Calvet, C. C and Grivetti, L. E. (2000). Energy and micronutrient composition of dietary and medicinal wild plants consumed during drought. Study of rural Fulani, Northeastern Nigeria, International Journal of food sciences and nutrition, 51 (3). 195-208.

Nivedita, T. M. A. (2017). Wild Plants of India- A review, International Journal of Academic Research, 4 (1). 189- 198.

Oak, G., Kurve, P., Kurve, S., and Pejaver, M. (2015). Ethno-botanical studies of edible plants used by tribal women of Thane District, Journal of Medicinal Plants Studies, 3(2) 90-94.

Ogle, B. M., Tuyet, H. O, , Duyet, H. N. and Dung, N. N. (2003). Food, feed or medicine: the multiple functions of edible wild plants in Vietnam, Economic Botany, 57(1) 103-117.

Padulosi, S., Heywood, V., Hunter, D. and Jarvis, A. (2011). Underutilized species and climate change: current status and outlook, In: Crop Adaptation to Climate Change, First Edition (Yadav SS, Redden RJ, Hatfield JL, Lotze-Campen $\mathrm{H}$ and Hall AE) (Blackwell Publishing Directorate), 507-521.

Panda, T. (2014). Traditional knowledge on wild edible plants as livelihood food in Odisha, India, Journal of Biology and Earth Sciences, 4(2), 144-159.

Patil, M. V. and Patil, D. A. (2005). Ethnomedicinal practices of Nasik district, Maharashtra, Indian Journal of Traditional Knowledge, 4(3), 287- 290.

Pieroni, A. (2000). Medicinal plants and food medicines in the folk traditions of the upper Lucca Province, Italy, Journal of Ethnopharmacology, 70(3). 235-273.

Samant, S. (1997). Diversity, Endemism and economic potential of wild edible plants of Indian Himalaya, The International Journal of Sustainable Development and World Ecology, 4(3). 179-191.

Sharma, P. P. and Mujumdar, A. M .(2003). Traditional knowledge on plants from Toranmal Plateau of Maharashtra, Indian Journal of Traditional Knowledge, 2 (3), 292- 296.

Shivprasad, M., Rane, M. and Satavi, V. (2016). Documentation and Ethnobotanical survey of wild edible plants from Palghar district, Asian Journal of Pharmaceuticals and Clinical Research, 9(2) 16- 19.

Singh, B., Sinha, B. K., Phunkan, S., Borthakur, S. and Singh, V. (2012), Wild edible plants used by Garo tribes of Nokrek Biosphere Reserve in Meghalaya, India. Indian Journal of Traditional knowledge, 11 (1) $166-171$.

Sonawane V. B, Saler R. S, Sonawane M. D and Kadam V. B (2012). Ethnobotanical Studies of Mokhada, District Thane, International Journal of Life Science and Pharma Research, 2 (2). 88- 93. 


\begin{tabular}{|c|c|c|c|c|c|c|c|c|c|}
\hline $\begin{array}{l}\text { Sr. } \\
\text { No }\end{array}$ & $\begin{array}{l}\text { Local } \\
\text { Name of } \\
\text { Species }\end{array}$ & $\begin{array}{l}\text { Scientific } \\
\text { Name }\end{array}$ & Family & Habit & $\begin{array}{l}\text { Season } \\
\text { of availa- } \\
\text { bility }\end{array}$ & $\begin{array}{l}\text { Plant } \\
\text { part } \\
\text { used }\end{array}$ & $\begin{array}{l}\text { Signifi- } \\
\text { cance }\end{array}$ & $\begin{array}{l}\text { Recipes } \\
\text { (If any) }\end{array}$ & $\begin{array}{l}\text { Medicinal } \\
\text { information }\end{array}$ \\
\hline 1. & $\begin{array}{l}\text { Aagheda/ } \\
\text { Achira }\end{array}$ & $\begin{array}{l}\text { Achyranthes- } \\
\text { aspera L. }\end{array}$ & $\begin{array}{l}\text { Amaranth- } \\
\text { aceae }\end{array}$ & Herb & Monsoon & $\begin{array}{l}\text { Tender } \\
\text { leaves }\end{array}$ & $\begin{array}{l}\text { Food } \\
\text { and } \\
\text { Medicinal }\end{array}$ & $\begin{array}{l}\text { Tender leaves are soaked in } \\
\text { water and cooked with onion } \\
\text { and local masalas as a } \\
\text { vegetable. }\end{array}$ & $\begin{array}{l}\text { Root powder } \\
\text { extract is con- } \\
\text { sumed during } \\
\text { tooth ache. }\end{array}$ \\
\hline 2. & $\begin{array}{l}\text { Aalambi/ } \\
\text { Alimbi }\end{array}$ & $\begin{array}{l}\text { Pleurotus } \\
\text { sps }\end{array}$ & $\begin{array}{l}\text { Agarica- } \\
\text { ceae }\end{array}$ & Fungus & Monsoon & $\begin{array}{l}\text { Whole } \\
\text { mushroom }\end{array}$ & Food & $\begin{array}{l}\text { Mushrooms are boiled and } \\
\text { cooked with local spices. }\end{array}$ & - \\
\hline 3. & $\begin{array}{l}\text { Abay/ } \\
\text { Abai/ } \\
\text { Chopdi }\end{array}$ & $\begin{array}{l}\text { Canavalia } \\
\text { gladiata } \\
\text { (Jacq.) DC. }\end{array}$ & $\begin{array}{l}\text { Legumino- } \\
\text { sae }\end{array}$ & Climber & Monsoon & Pods & Food & $\begin{array}{l}\text { The young pods are boiled } \\
\text { and are cooked with onion } \\
\text { and chilly. The excess } \\
\text { boiled water is drained. }\end{array}$ & - \\
\hline 4. & Adulsa & $\begin{array}{l}\text { Justicia } \\
\text { adhatoda L. }\end{array}$ & $\begin{array}{l}\text { Acantha- } \\
\text { ceae }\end{array}$ & Shrub & $\begin{array}{l}\text { All } \\
\text { season }\end{array}$ & Leaves & Medicinal & - & $\begin{array}{l}\text { Decoction of } \\
\text { leaves is con- } \\
\text { sumed during } \\
\text { cough. }\end{array}$ \\
\hline 5. & $\begin{array}{l}\text { Akri/ } \\
\text { Akkad- } \\
\text { ghad }\end{array}$ & $\begin{array}{l}\text { Athyrium } \\
\text { hohenacke- } \\
\text { rianum } \\
\text { T. Moore }\end{array}$ & $\begin{array}{l}\text { Athyria- } \\
\text { ceae }\end{array}$ & Fern & Monsoon & $\begin{array}{l}\text { Tender } \\
\text { shoots }\end{array}$ & Food & $\begin{array}{l}\text { Young shoots are boiled and } \\
\text { cooked as vegetable in local } \\
\text { spices. }\end{array}$ & - \\
\hline 6. & Aliv/Alav & $\begin{array}{l}\text { Meyna } \\
\text { laxiflora } \\
\text { Robyns }\end{array}$ & Rubiaceae & Tree & Monsoon & Fruits & $\begin{array}{l}\text { Food } \\
\text { (Sold in } \\
\text { the market } \\
\text { during } \\
\text { season) }\end{array}$ & $\begin{array}{l}\text { Directly } \\
\text { consumption of } \\
\text { fruits. }\end{array}$ & - \\
\hline 7. & $\begin{array}{l}\text { Alu/ } \\
\text { Arabi/ } \\
\text { Tera/Teri }\end{array}$ & $\begin{array}{l}\text { Colocasia } \\
\text { esculenta }(\mathrm{L} .) \\
\text { Schott }\end{array}$ & Araceae & Herb & Monsoon & $\begin{array}{l}\text { Leaves } \\
\text { and } \\
\text { Tuber }\end{array}$ & Food & $\begin{array}{l}\text { 1. Leaves are steamed and } \\
\text { layered with gram flour to } \\
\text { make local dish called } \\
\text { 'Wadi'. }\end{array}$ & - \\
\hline
\end{tabular}




\begin{tabular}{|c|c|c|c|c|c|c|c|c|c|}
\hline $\begin{array}{l}\text { Sr. } \\
\text { No }\end{array}$ & $\begin{array}{l}\text { Local } \\
\text { Name of } \\
\text { Species }\end{array}$ & $\begin{array}{l}\text { Scientific } \\
\text { Name }\end{array}$ & Family & Habit & $\begin{array}{l}\text { Season } \\
\text { of availa- } \\
\text { bility }\end{array}$ & $\begin{array}{l}\text { Plant } \\
\text { part } \\
\text { used }\end{array}$ & $\begin{array}{l}\text { Signifi- } \\
\text { cance }\end{array}$ & $\begin{array}{l}\text { Recipes } \\
\text { (If any) }\end{array}$ & $\begin{array}{l}\text { Medicinal } \\
\text { information }\end{array}$ \\
\hline & & & & & & & & $\begin{array}{l}\text { 2. Green curry or 'Saag' is } \\
\text { also prepared by using the } \\
\text { leaves. } \\
\text { 3. Tubers are boiled and } \\
\text { cooked as vegetable. }\end{array}$ & \\
\hline 8. & Amba & $\begin{array}{l}\text { Mangifera } \\
\text { indicaL. }\end{array}$ & $\begin{array}{l}\text { Anacardia- } \\
\text { ceae }\end{array}$ & Tree & Summer & $\begin{array}{l}\text { Bark and } \\
\text { Fruits }\end{array}$ & $\begin{array}{l}\text { Food } \\
\text { and } \\
\text { Medicinal }\end{array}$ & $\begin{array}{l}\text { Directly consumption of } \\
\text { fruits }\end{array}$ & $\begin{array}{l}\text { Bark decoction is } \\
\text { consumed during } \\
\text { stomach ache }\end{array}$ \\
\hline 9. & Ambadi & $\begin{array}{l}\text { Hibiscus } \\
\text { sabdariffa.L. }\end{array}$ & Malvaceae & Herb & Monsoon & $\begin{array}{l}\text { Leaves } \\
\text { and Fruits }\end{array}$ & $\begin{array}{l}\text { Food } \\
\text { and } \\
\text { Medicinal }\end{array}$ & $\begin{array}{l}\text { 1. Initially leaves are dried } \\
\text { and cooked as vegetable } \\
\text { using local masalas. } \\
\text { 2. As it is sour to taste only } \\
\text { small portion is added just } \\
\text { to add taste while cooking } \\
\text { curry or fish. }\end{array}$ & $\begin{array}{l}\text { A small portion } \\
\text { of the dried fruit } \\
\text { is boiled and the } \\
\text { water is con- } \\
\text { sumed to cure } \\
\text { cough or stom- } \\
\text { ach infection. }\end{array}$ \\
\hline 10. & Ambuti & $\begin{array}{l}\text { Begonia } \\
\text { crenata } \\
\text { Dryand }\end{array}$ & $\begin{array}{l}\text { Begonia- } \\
\text { ceae }\end{array}$ & Herb & Monsoon & Leaves & Food & $\begin{array}{l}\text { Leaves are consumed } \\
\text { directly or added in } \\
\text { vegetable for its sour taste. }\end{array}$ & - \\
\hline 11. & Asand & $\begin{array}{l}\text { Bridelia } \\
\text { retusa }(\mathrm{L} .) \\
\text { A.Juss. }\end{array}$ & $\begin{array}{l}\text { Phylla- } \\
\text { nthaceae }\end{array}$ & Tree & Monsoon & $\begin{array}{l}\text { Fruits } \\
\text { and } \\
\text { Bark }\end{array}$ & $\begin{array}{l}\text { Food } \\
\text { and } \\
\text { Medicinal }\end{array}$ & $\begin{array}{l}\text { Directly consumption of } \\
\text { ripe fruits }\end{array}$ & $\begin{array}{l}\text { Bark extract is } \\
\text { consumed } \\
\text { during ear ache. } \\
\text { Also effective on } \\
\text { stomach ache. }\end{array}$ \\
\hline 12. & Avala & $\begin{array}{l}\text { Phyllanthus } \\
\text { emblicaL }\end{array}$ & $\begin{array}{l}\text { Phyllan- } \\
\text { thaceae }\end{array}$ & Tree & Winter & $\begin{array}{l}\text { Fruits } \\
\text { and } \\
\text { Stem }\end{array}$ & $\begin{array}{l}\text { Food } \\
\text { and } \\
\text { Medicinal }\end{array}$ & $\begin{array}{l}\text { Ripe fruits are consumed } \\
\text { raw or pickeled through } \\
\text { sun drying. }\end{array}$ & $\begin{array}{l}\text { Stem extract is } \\
\text { consumed during } \\
\text { dental pain. }\end{array}$ \\
\hline 13. & Badade & $\begin{array}{l}\text { Arisaema } \\
\text { murrayi } \\
\text { (J. Graham) } \\
\text { Hook. }\end{array}$ & Araceae & Herb & Monsoon & Tuber & Food & $\begin{array}{l}\text { Tubers are soon boiled } \\
\text { and cooked and } \\
\text { consumed directly or } \\
\text { as vegetable. }\end{array}$ & - \\
\hline
\end{tabular}




\begin{tabular}{|c|c|c|c|c|c|c|c|c|c|}
\hline $\begin{array}{l}\text { Sr. } \\
\text { No }\end{array}$ & $\begin{array}{l}\text { Local } \\
\text { Name of } \\
\text { Species }\end{array}$ & $\begin{array}{l}\text { Scientific } \\
\text { Name }\end{array}$ & Family & Habit & $\begin{array}{l}\text { Season } \\
\text { of availa- } \\
\text { bility }\end{array}$ & $\begin{array}{l}\text { Plant } \\
\text { part } \\
\text { used }\end{array}$ & $\begin{array}{l}\text { Signifi- } \\
\text { cance }\end{array}$ & $\begin{array}{l}\text { Recipes } \\
\text { (If any) }\end{array}$ & $\begin{array}{l}\text { Medicinal } \\
\text { information }\end{array}$ \\
\hline 14. & $\begin{array}{l}\text { Bafali/ } \\
\text { Baphali }\end{array}$ & $\begin{array}{l}\text { Heracleum } \\
\text { grande } \\
\text { (Dalzell \& } \\
\text { A. Gibson) } \\
\text { Mukhop. }\end{array}$ & Apiaceae & Herb & Monsoon & $\begin{array}{l}\text { Leaves, } \\
\text { roots } \\
\text { and } \\
\text { seeds }\end{array}$ & $\begin{array}{l}\text { Food and } \\
\text { Medicinal } \\
\text { (Sold in } \\
\text { the market } \\
\text { during } \\
\text { season) }\end{array}$ & $\begin{array}{l}\text { 1. Steamed leaves are saute } \\
\text { on oil to make vegetable. } \\
\text { 2. Seeds are used in } \\
\text { preparation of sauce 'chatni'. }\end{array}$ & $\begin{array}{l}\text { Seed powder and } \\
\text { root decoction is } \\
\text { used to cure } \\
\text { stomach pain. }\end{array}$ \\
\hline 15. & Bahava & $\begin{array}{l}\text { Cassia } \\
\text { fistula L. }\end{array}$ & $\begin{array}{l}\text { Legu- } \\
\text { minosae }\end{array}$ & Tree & Summer & Flowers & Food & $\begin{array}{l}\text { Flowers are boiled and } \\
\text { cooked with onion and } \\
\text { chilies. The water is drained } \\
\text { after thoroughly after } \\
\text { cooking. }\end{array}$ & - \\
\hline 16. & $\begin{array}{l}\text { Bamboo/ } \\
\text { Vasate }\end{array}$ & $\begin{array}{l}\text { Bambusa } \\
\text { bambos(L.) } \\
\text { Voss }\end{array}$ & Poaceae & Herb & $\begin{array}{l}\text { Mon- } \\
\text { soon }\end{array}$ & Shoots & $\begin{array}{l}\text { Food } \\
\text { (Sold in } \\
\text { the market } \\
\text { during } \\
\text { season) }\end{array}$ & $\begin{array}{l}\text { Young shoots used to } \\
\text { prepare vegetable as } \\
\text { well as preserved in } \\
\text { the form of a pickle. }\end{array}$ & - \\
\hline 17. & Bandgul & $\begin{array}{l}\text { Dendro- } \\
\text { phthoe } \\
\text { falcata(L.f.) } \\
\text { Ettingsh. }\end{array}$ & $\begin{array}{l}\text { Lorantha- } \\
\text { ceae }\end{array}$ & Shrub & Winter & Fruits & Food & Fruits are consumed directly. & - \\
\hline 18. & Beheda & $\begin{array}{l}\text { Terminalia } \\
\text { bellirica } \\
\text { (Gaertn.) } \\
\text { Roxb. }\end{array}$ & $\begin{array}{l}\text { Combreta- } \\
\text { ceae }\end{array}$ & Tree & Monsoon & $\begin{array}{l}\text { Fruits } \\
\text { and } \\
\text { Seeds }\end{array}$ & $\begin{array}{l}\text { Food } \\
\text { and } \\
\text { Medicinal }\end{array}$ & $\begin{array}{l}\text { Seeds consumed raw by } \\
\text { children only after the fruit } \\
\text { dries and breaks. Too much } \\
\text { consumption of seeds may } \\
\text { cause giddiness in children. }\end{array}$ & $\begin{array}{l}\text { Powder of fruits } \\
\text { is consumed } \\
\text { during cough. }\end{array}$ \\
\hline 19. & Bel & $\begin{array}{l}\text { Aegle } \\
\text { marmelos } \\
\text { (L.) Corrêa }\end{array}$ & Rutaceae & Tree & Summer & $\begin{array}{l}\text { Fruits } \\
\text { and } \\
\text { Bark }\end{array}$ & $\begin{array}{l}\text { Food } \\
\text { and } \\
\text { Medicinal }\end{array}$ & $\begin{array}{l}\text { Ripe fruits are directly } \\
\text { consumed or added in juice } \\
\text { 'Sharbat' }\end{array}$ & $\begin{array}{l}\text { Bark decoction } \\
\text { consumed during } \\
\text { dental pain. }\end{array}$ \\
\hline
\end{tabular}




\begin{tabular}{|c|c|c|c|c|c|c|c|c|c|}
\hline $\begin{array}{l}\text { Sr. } \\
\text { No }\end{array}$ & $\begin{array}{l}\text { Local } \\
\text { Name of } \\
\text { Species }\end{array}$ & $\begin{array}{l}\text { Scientific } \\
\text { Name }\end{array}$ & Family & Habit & $\begin{array}{l}\text { Season } \\
\text { of availa- } \\
\text { bility }\end{array}$ & $\begin{array}{l}\text { Plant } \\
\text { part } \\
\text { used }\end{array}$ & $\begin{array}{l}\text { Signifi- } \\
\text { cance }\end{array}$ & $\begin{array}{l}\text { Recipes } \\
\text { (If any) }\end{array}$ & $\begin{array}{l}\text { Medicinal } \\
\text { information }\end{array}$ \\
\hline 20. & $\begin{array}{l}\text { Bhagar/ } \\
\text { Varai }\end{array}$ & $\begin{array}{l}\text { Panicum } \\
\text { miliaceum L. }\end{array}$ & Poaceae & Herb & Winter & Seeds & Food & $\begin{array}{l}\text { 1. Mostly consumed by } \\
\text { preparing Soup } \\
\text { 2. Grains are boiled to } \\
\text { prepare 'Khichadi' (Type of } \\
\text { rice item) by adding local } \\
\text { ingredients. }\end{array}$ & - \\
\hline 21. & Bharangi & $\begin{array}{l}\text { Rotheca } \\
\text { serrata (L.) } \\
\text { Steane \& } \\
\text { Mabb. }\end{array}$ & Lamiaceae & Shrub & Monsoon & Leaves & Food & $\begin{array}{l}\text { Young leaves are used to } \\
\text { make vegetable. }\end{array}$ & - \\
\hline 22. & Bhokar & $\begin{array}{l}\text { Cordia } \\
\text { dichotoma } \\
\text { G.Forst. }\end{array}$ & Boraginacea & e Tree & Summer & Fruits & Food & $\begin{array}{l}\text { Raw fruits are pickled while } \\
\text { ripe fruits are directly } \\
\text { consumed }\end{array}$ & - \\
\hline 23. & $\begin{array}{l}\text { Bhopla/ } \\
\text { Bhopala/ } \\
\text { Dangar/ } \\
\text { Dongar- } \\
\text { bhaji }\end{array}$ & $\begin{array}{l}\text { Cucurbita } \\
\text { maxima } \\
\text { Duchesne }\end{array}$ & $\begin{array}{l}\text { Cucurbita- } \\
\text { ceae }\end{array}$ & Climber & Monsoon & Fruits & Food & $\begin{array}{l}\text { Fruits are boiled and cookec } \\
\text { as a vegetable. }\end{array}$ & - \\
\hline 24. & Bibba & $\begin{array}{l}\text { Semecarpus } \\
\text { anacardium } \mathrm{L}\end{array}$ & $\begin{array}{l}\text { Anacardia- } \\
\text { ceae }\end{array}$ & Tree & Summer & $\begin{array}{l}\text { Fruits and } \\
\text { Seeds }\end{array}$ & Food & $\begin{array}{l}\text { 1. Fleshy part of fruit is } \\
\text { consumed directly. } \\
\text { 2. Seeds known as Godambi } \\
\text { are consumed raw. }\end{array}$ & - \\
\hline 25. & $\begin{array}{l}\text { Bivala / } \\
\text { Beeja }\end{array}$ & $\begin{array}{l}\text { Pterocarpus- } \\
\text { marsupium } \\
\text { Roxb }\end{array}$ & $\begin{array}{l}\text { Legumino- } \\
\text { sae }\end{array}$ & Tree & All season & $\begin{array}{l}\text { Leaves } \\
\text { and Bark }\end{array}$ & $\begin{array}{l}\text { Food and } \\
\text { Medicinal }\end{array}$ & $\begin{array}{l}\text { Young leaves boiled and } \\
\text { cooked used as vegetable }\end{array}$ & $\begin{array}{l}\text { Bark powder } \\
\text { used to cure } \\
\text { dental problem }\end{array}$ \\
\hline 26. & Bondara & $\begin{array}{l}\text { Lagerstrom- } \\
\text { ea parviflora } \\
\text { Roxb. }\end{array}$ & $\begin{array}{l}\text { Lythara- } \\
\text { ceae }\end{array}$ & Tree & All season & Leaves & Food & $\begin{array}{l}\text { Tender leaves are mixed with } \\
\text { other wild vegetable and } \\
\text { cooked to remove itchiness } \\
\text { occurring in throat. }\end{array}$ & - \\
\hline
\end{tabular}




\begin{tabular}{|c|c|c|c|c|c|c|c|c|c|}
\hline $\begin{array}{l}\text { Sr. } \\
\text { No }\end{array}$ & $\begin{array}{l}\text { Local } \\
\text { Name of } \\
\text { Species }\end{array}$ & $\begin{array}{l}\text { Scientific } \\
\text { Name }\end{array}$ & Family & Habit & $\begin{array}{l}\text { Season } \\
\text { of availa- } \\
\text { bility }\end{array}$ & $\begin{array}{l}\text { Plant } \\
\text { part } \\
\text { used }\end{array}$ & $\begin{array}{l}\text { Signifi- } \\
\text { cance }\end{array}$ & $\begin{array}{l}\text { Recipes } \\
\text { (If any) }\end{array}$ & $\begin{array}{l}\text { Medicinal } \\
\text { information }\end{array}$ \\
\hline 27. & Bor & $\begin{array}{l}\text { Ziziphus } \\
\text { jujube Mill. }\end{array}$ & $\begin{array}{l}\text { Rhamna- } \\
\text { ceae }\end{array}$ & Tree & Winter & Fruits & Food & Direct consumption of fruits. & - \\
\hline 28. & Borod & $\begin{array}{l}\text { Echino- } \\
\text { chloa colona } \\
\text { (L.) Link }\end{array}$ & Poaceae & Herb & Monsoon & Seeds & Food & $\begin{array}{l}\text { Seeds are used in the } \\
\text { preparation of sweets. }\end{array}$ & - \\
\hline 29. & $\begin{array}{l}\text { Chaivel/ } \\
\text { Chaikand/ } \\
\text { Chayavel }\end{array}$ & $\begin{array}{l}\text { Dioscorea } \\
\text { alataL. }\end{array}$ & $\begin{array}{l}\text { Dioscorea- } \\
\text { ceae }\end{array}$ & Climber & Monsoon & Tubers & Food & $\begin{array}{l}\text { Tubers are boiled, cooked } \\
\text { and consumed as vegetable. }\end{array}$ & - \\
\hline 30. & Chavali & $\begin{array}{l}\text { Vigna } \\
\text { unguiculata } \\
\text { (L.) walp. }\end{array}$ & $\begin{array}{l}\text { Legumino- } \\
\text { sae }\end{array}$ & Climber & Monsoon & Seeds & Food & $\begin{array}{l}\text { Seeds are boiled and cooked } \\
\text { as vegetable. }\end{array}$ & - \\
\hline 31. & $\begin{array}{l}\text { Chibud/ } \\
\text { Musk- } \\
\text { melon }\end{array}$ & $\begin{array}{l}\text { Cucumis } \\
\text { melo L. }\end{array}$ & $\begin{array}{l}\text { Cucurbita- } \\
\text { ceae }\end{array}$ & Climber & Summer & Fruit & Food & $\begin{array}{l}\text { Fruits are consumed directly } \\
\text { on ripening. }\end{array}$ & - \\
\hline 32. & Chinch & $\begin{array}{l}\text { Tamarindus } \\
\text { indica L. }\end{array}$ & $\begin{array}{l}\text { Legumino- } \\
\text { sae }\end{array}$ & Tree & Summer & Ripe fruits & Food & $\begin{array}{l}\text { 1. Ripe fruit are added in } \\
\text { vegetables to add the sour } \\
\text { flavor. } \\
\text { 2. Riped fruits are also } \\
\text { consumed directly. }\end{array}$ & - \\
\hline 33. & Chirambot & $\begin{array}{l}\text { Physalis } \\
\text { minima L. }\end{array}$ & Solanaceae & Herb & Monsoon & Fruits & Food & $\begin{array}{l}\text { Ripe fruits are consumed } \\
\text { directly. }\end{array}$ & - \\
\hline 34. & Chirati & $\begin{array}{l}\text { Mukia } \\
\text { madera- } \\
\text { spatana }(\mathrm{L} .) \\
\text { M.Roem. }\end{array}$ & $\begin{array}{l}\text { Cucurbita- } \\
\text { ceae }\end{array}$ & Climber & Monsoon & Fruits & Food & $\begin{array}{l}\text { Ripe fruits are consumed } \\
\text { directly. }\end{array}$ & - \\
\hline 35. & $\begin{array}{l}\text { Chunch/ } \\
\text { Chuch/ } \\
\text { Chinch- } \\
\text { nuk }\end{array}$ & $\begin{array}{l}\text { Corchorus } \\
\text { olitorius. L }\end{array}$ & Malvaceae & Herb & Monsoon & Leaves & Food & $\begin{array}{l}\text { Young leaves are boiled and } \\
\text { cooked as vegetable. }\end{array}$ & - \\
\hline
\end{tabular}




\begin{tabular}{|c|c|c|c|c|c|c|c|c|c|}
\hline $\begin{array}{l}\text { Sr. } \\
\text { No }\end{array}$ & $\begin{array}{l}\text { Local } \\
\text { Name of } \\
\text { Species }\end{array}$ & $\begin{array}{l}\text { Scientific } \\
\text { Name }\end{array}$ & Family & Habit & $\begin{array}{l}\text { Season } \\
\text { of availa- } \\
\text { bility }\end{array}$ & $\begin{array}{l}\text { Plant } \\
\text { part } \\
\text { used }\end{array}$ & $\begin{array}{l}\text { Signifi- } \\
\text { cance }\end{array}$ & $\begin{array}{l}\text { Recipes } \\
\text { (If any) }\end{array}$ & $\begin{array}{l}\text { Medicinal } \\
\text { information }\end{array}$ \\
\hline 36. & Dauda & $\begin{array}{l}\text { Sterculia } \\
\text { gutata } \\
\text { Roxb. ex } \\
\text { G.Don }\end{array}$ & Malvaceae & Tree & Winter & Seeds & Food & $\begin{array}{l}\text { Roasted seeds are consumed } \\
\text { directly. }\end{array}$ & - \\
\hline 37. & $\begin{array}{l}\text { Dehagadi/ } \\
\text { Digha- } \\
\text { vadi/ } \\
\text { Konchi }\end{array}$ & $\begin{array}{l}\text { Dioscorea } \\
\text { wallichii } \\
\text { Hook.f. }\end{array}$ & $\begin{array}{l}\text { Dioscorea- } \\
\text { ceae }\end{array}$ & Climber & Monsoon & $\begin{array}{l}\text { Tender } \\
\text { leaves } \\
\text { and } \\
\text { shoots }\end{array}$ & Food & $\begin{array}{l}\text { Tender leaves and shoots are } \\
\text { boiled and cooked with local } \\
\text { masalas to be consumed as } \\
\text { vegetable. }\end{array}$ & - \\
\hline 38. & $\begin{array}{l}\text { Dhavda/ } \\
\text { Dhamoda }\end{array}$ & $\begin{array}{l}\text { Anogeissus } \\
\text { latifolia } \\
\text { (Roxb. ex } \\
\text { DC.) Wall. } \\
\text { ex Guillem. } \\
\& \text { Perr. }\end{array}$ & $\begin{array}{l}\text { Combreta- } \\
\text { ceae }\end{array}$ & Tree & $\begin{array}{l}\text { All } \\
\text { season }\end{array}$ & Gum & Food & $\begin{array}{l}\text { Gum is edible and added in } \\
\text { various curries and } \\
\text { vegetables. }\end{array}$ & - \\
\hline 39. & Dhayati & $\begin{array}{l}\text { Woodfordia } \\
\text { fruticosa(L.) } \\
\text { Kurz }\end{array}$ & Lytharaceae & Shrub & Winter & Flowers & Food & $\begin{array}{l}\text { Flowers are not only used to } \\
\text { extract nectar but they are } \\
\text { also cooked and consumed. }\end{array}$ & - \\
\hline 40. & Dudhi & $\begin{array}{l}\text { Lagenaria } \\
\text { siceraria } \\
\text { (Molina) } \\
\text { Standl. }\end{array}$ & $\begin{array}{l}\text { Cucurbita- } \\
\text { ceae }\end{array}$ & Climber & Monsoon & Fruits & Food & $\begin{array}{l}\text { Fruits are boiled and cooked } \\
\text { as a vegetable. }\end{array}$ & - \\
\hline 41. & Erand & $\begin{array}{l}\text { Ricinus } \\
\text { communis L. }\end{array}$ & $\begin{array}{l}\text { Euphorbia- } \\
\text { ceae }\end{array}$ & Shrub & All season & Seeds & Medicinal & - & $\begin{array}{l}\text { Oil is extracted } \\
\text { from the seeds of } \\
\text { Erand which are } \\
\text { used as laxative } \\
\text { during stomach } \\
\text { problems. }\end{array}$ \\
\hline 42. & Fanas & $\begin{array}{l}\text { Artocarpus } \\
\text { hetero- } \\
\text { phyllus } \\
\text { Lam. }\end{array}$ & Moraceae & Tree & Summer & Fruits & Food & Direct consumption of fruits. & - \\
\hline
\end{tabular}




\begin{tabular}{|c|c|c|c|c|c|c|c|c|c|}
\hline $\begin{array}{l}\text { Sr. } \\
\text { No }\end{array}$ & $\begin{array}{l}\text { Local } \\
\text { Name of } \\
\text { Species }\end{array}$ & $\begin{array}{l}\text { Scientific } \\
\text { Name }\end{array}$ & Family & Habit & $\begin{array}{l}\text { Season } \\
\text { of availa- } \\
\text { bility }\end{array}$ & $\begin{array}{l}\text { Plant } \\
\text { part } \\
\text { used }\end{array}$ & $\begin{array}{l}\text { Signifi- } \\
\text { cance }\end{array}$ & $\begin{array}{l}\text { Recipes } \\
\text { (If any) }\end{array}$ & $\begin{array}{l}\text { Medicinal } \\
\text { information }\end{array}$ \\
\hline 43. & $\begin{array}{l}\text { Fatan- } \\
\text { gadi/ } \\
\text { Phan- } \\
\text { gota/ } \\
\text { Phata- } \\
\text { ngdi/ } \\
\text { Amba- } \\
\text { ttingra }\end{array}$ & $\begin{array}{l}\text { Embelia } \\
\text { tsjerium- } \\
\text { cottam } \\
\text { (Roem. } \\
\text { \& Schult.) } \\
\text { A.DC. }\end{array}$ & $\begin{array}{l}\text { Primula- } \\
\text { ceae }\end{array}$ & Shrub & $\begin{array}{l}\text { All } \\
\text { season }\end{array}$ & $\begin{array}{l}\text { Leaves } \\
\text { and Seeds }\end{array}$ & $\begin{array}{l}\text { Food and } \\
\text { Medicinal }\end{array}$ & $\begin{array}{l}\text { Leaves are boiled and } \\
\text { cooked as vegetable using } \\
\text { local masalas. }\end{array}$ & $\begin{array}{l}\text { Seeds are con- } \\
\text { sumed during } \\
\text { stomach pain. }\end{array}$ \\
\hline 44. & Gagola & $\begin{array}{l}\text { Ipomoea } \\
\text { muricata } \\
\text { (L.) Jacq. }\end{array}$ & $\begin{array}{l}\text { Convolvu- } \\
\text { laceae }\end{array}$ & Climber & Monsoon & Fruits & Food & $\begin{array}{l}\text { Fruits are consumed only } \\
\text { after removal of the seed and } \\
\text { stir fried with onion and } \\
\text { local masalas to make } \\
\text { vegetable. }\end{array}$ & \\
\hline 45. & $\begin{array}{l}\text { Gala/ } \\
\text { Gela } \\
\text { Ghela/ } \\
\text { Gal }\end{array}$ & $\begin{array}{l}\text { Catunare- } \\
\text { gam spinosa } \\
\text { (Thunb.) } \\
\text { Tirveng. }\end{array}$ & Rubiaceae & Tree & Monsoon & $\begin{array}{l}\text { Tender } \\
\text { fruits }\end{array}$ & Food & $\begin{array}{l}\text { Tender fruits are boiled and } \\
\text { cooked in niger seeds and } \\
\text { local masala to be consumed } \\
\text { as vegetable. }\end{array}$ & \\
\hline 46. & $\begin{array}{l}\text { Gar- } \\
\text { bhanda }\end{array}$ & $\begin{array}{l}\text { Leea } \\
\text { asiatica(L.) } \\
\text { Ridsdale }\end{array}$ & Vitaceae & Shrub & Monsoon & Leaves & Food & $\begin{array}{l}\text { This wild edible was } \\
\text { recorded in Jawhar but the } \\
\text { tribes do not consume the } \\
\text { same. In other parts of } \\
\text { Maharashtra, the leaves are } \\
\text { cooked and consumed. }\end{array}$ & \\
\hline 47. & Garbhend & $\begin{array}{l}\text { Thespesia } \\
\text { lampas } \\
\text { (Cav.) } \\
\text { Dalzell }\end{array}$ & Malvaceae & Shrub & Monsoon & Roots & Medicinal & - & $\begin{array}{l}\text { The roots decoc- } \\
\text { tion is consumed } \\
\text { during yellow } \\
\text { urination. }\end{array}$ \\
\hline 48. & Gavar & $\begin{array}{l}\text { Cyamopsis } \\
\text { tetragono- } \\
\text { loba }(\mathrm{L} .) \\
\text { Taub. }\end{array}$ & $\begin{array}{l}\text { Legumino- } \\
\text { sae }\end{array}$ & Shrub & Winter & Fruits & Food & $\begin{array}{l}\text { Fruits are boiled and cooked } \\
\text { as vegetable. }\end{array}$ & - \\
\hline
\end{tabular}




\begin{tabular}{|c|c|c|c|c|c|c|c|c|c|}
\hline $\begin{array}{l}\text { Sr. } \\
\text { No }\end{array}$ & $\begin{array}{l}\text { Local } \\
\text { Name of } \\
\text { Species }\end{array}$ & $\begin{array}{l}\text { Scientific } \\
\text { Name }\end{array}$ & Family & Habit & $\begin{array}{l}\text { Season } \\
\text { of availa- } \\
\text { bility }\end{array}$ & $\begin{array}{l}\text { Plant } \\
\text { part } \\
\text { used }\end{array}$ & $\begin{array}{l}\text { Signifi- } \\
\text { cance }\end{array}$ & $\begin{array}{l}\text { Recipes } \\
\text { (If any) }\end{array}$ & $\begin{array}{l}\text { Medicinal } \\
\text { information }\end{array}$ \\
\hline 49. & $\begin{array}{l}\text { Gavathi- } \\
\text { udid }\end{array}$ & $\begin{array}{l}\text { Vigna } \\
\text { mungo(L.) } \\
\text { Hepper }\end{array}$ & $\begin{array}{l}\text { Legumino- } \\
\text { sae }\end{array}$ & Herb & Monsoon & Seeds & Food & $\begin{array}{l}\text { Seeds are cooked in the form } \\
\text { of curry or vegetable. }\end{array}$ & - \\
\hline 50. & Gholu & $\begin{array}{l}\text { Portulaca } \\
\text { oleracea } \mathrm{L}\end{array}$ & $\begin{array}{l}\text { Portulaca- } \\
\text { ceae }\end{array}$ & Herb & Monsoon & Leaves & Food & $\begin{array}{l}\text { Leaves are boiled and cooked } \\
\text { as vegetable. }\end{array}$ & - \\
\hline 51. & $\begin{array}{l}\text { Ghugur- } \\
\text { val }\end{array}$ & $\begin{array}{l}\text { Flacaurtia } \\
\text { indica } \\
\text { (Burm.f.) } \\
\text { Merr. }\end{array}$ & Salicaceae & Tree & Monsoon & Fruits & Food & Direct consumption of fruits. & - \\
\hline 52. & Girjala & $\begin{array}{l}\text { Marsilea } \\
\text { quadrifolia L. }\end{array}$ & $\begin{array}{l}\text { Marsilea- } \\
\text { ceae }\end{array}$ & Herb & Winter & Leaves & Food & $\begin{array}{l}\text { Leaves are boiled and cooked } \\
\text { as vegetable. }\end{array}$ & - \\
\hline 53. & $\begin{array}{l}\text { God } \\
\text { Kanda/ } \\
\text { Karanda }\end{array}$ & $\begin{array}{l}\text { Dioscorea } \\
\text { esculenta } \\
\text { (Lour.) } \\
\text { Burkill }\end{array}$ & $\begin{array}{l}\text { Dioscorea- } \\
\text { ceae }\end{array}$ & Climber & Monsoon & $\begin{array}{l}\text { Bulbil and } \\
\text { Tuber }\end{array}$ & $\begin{array}{l}\text { Food } \\
\text { (Sold in } \\
\text { the market } \\
\text { during } \\
\text { season) }\end{array}$ & $\begin{array}{l}\text { Bulbils and tubers are boiled } \\
\text { and cooked as vegetable. }\end{array}$ & - \\
\hline 54. & Gometi & $\begin{array}{l}\text { Solena } \\
\text { amplexi- } \\
\text { caulis(Lam.) } \\
\text { Gandhi }\end{array}$ & $\begin{array}{l}\text { Cucurbita- } \\
\text { ceae }\end{array}$ & Climber & Monsoon & Tuber & Medicinal & - & $\begin{array}{l}\text { Tuber powder is } \\
\text { consumed to } \\
\text { increase the } \\
\text { appetite in } \\
\text { children. }\end{array}$ \\
\hline 55. & Gulvel & $\begin{array}{l}\text { Tinospora } \\
\text { sinensis } \\
\text { (Lour.) } \\
\text { Merr. }\end{array}$ & $\begin{array}{l}\text { Menisper- } \\
\text { maceae }\end{array}$ & Climber & Monsoon & Stem & Medicinal & - & $\begin{array}{l}\text { Stem decoction is } \\
\text { consumed during } \\
\text { acidity and heat } \\
\text { problem. }\end{array}$ \\
\hline 56. & Gunj & $\begin{array}{l}\text { Abrus } \\
\text { precatorius } \\
\text { L. }\end{array}$ & $\begin{array}{l}\text { Legumino- } \\
\text { sae }\end{array}$ & Climber & Monsoon & Leaves & $\begin{array}{l}\text { Food and } \\
\text { Medicinal }\end{array}$ & $\begin{array}{l}\text { Leaves are directly consumed } \\
\text { after meal or add in 'Pan' }\end{array}$ & $\begin{array}{l}\text { Leaves are used } \\
\text { as medicine on } \\
\text { cough and it also } \\
\text { helps in } \\
\text { digestion }\end{array}$ \\
\hline
\end{tabular}




\begin{tabular}{|c|c|c|c|c|c|c|c|c|c|}
\hline $\begin{array}{l}\text { Sr. } \\
\text { No }\end{array}$ & $\begin{array}{l}\text { Local } \\
\text { Name of } \\
\text { Species }\end{array}$ & $\begin{array}{l}\text { Scientific } \\
\text { Name }\end{array}$ & Family & Habit & $\begin{array}{l}\text { Season } \\
\text { of availa- } \\
\text { bility }\end{array}$ & $\begin{array}{l}\text { Plant } \\
\text { part } \\
\text { used }\end{array}$ & $\begin{array}{l}\text { Signifi- } \\
\text { cance }\end{array}$ & $\begin{array}{l}\text { Recipes } \\
\text { (If any) }\end{array}$ & $\begin{array}{l}\text { Medicinal } \\
\text { information }\end{array}$ \\
\hline 57. & Halad & $\begin{array}{l}\text { Curcuma } \\
\text { longa L. }\end{array}$ & $\begin{array}{l}\text { Zingibera- } \\
\text { ceae }\end{array}$ & Herb & $\begin{array}{l}\text { All } \\
\text { season }\end{array}$ & Rhizome & Food & $\begin{array}{l}\text { Rhizome powder is used in } \\
\text { daily cooking. }\end{array}$ & - \\
\hline 58. & Halinda & $\begin{array}{l}\text { Vigna } \\
\text { vexillata(L.) } \\
\text { A.Rich. }\end{array}$ & $\begin{array}{l}\text { Legumino- } \\
\text { sae }\end{array}$ & Climber & $\begin{array}{l}\text { All } \\
\text { season }\end{array}$ & Tuber & Food & $\begin{array}{l}\text { Tuber is consumed raw or is } \\
\text { boiled and cooked as } \\
\text { vegetable. }\end{array}$ & - \\
\hline 59. & Hirda & $\begin{array}{l}\text { Terminalia } \\
\text { chebula } \\
\text { Retz. }\end{array}$ & $\begin{array}{l}\text { Combreta- } \\
\text { ceae }\end{array}$ & Tree & Monsoon & Fruits & Medicinal & - & $\begin{array}{l}\text { Fruit powder is } \\
\text { consumed } \\
\text { against cough. }\end{array}$ \\
\hline 60. & Humb & $\begin{array}{l}\text { Miliusa } \\
\text { tomentosa } \\
\text { (Roxb.) } \\
\text { J.Sinclair }\end{array}$ & $\begin{array}{l}\text { Annona- } \\
\text { ceae }\end{array}$ & Tree & Summer & Fruits & Food & $\begin{array}{l}\text { Ripe fruits are consumed } \\
\text { directly. }\end{array}$ & - \\
\hline 61. & Jambhul & $\begin{array}{l}\text { Syzygium } \\
\text { cumini(L.) } \\
\text { Skeels }\end{array}$ & Myrtaceae & Tree & Summer & Fruits & Food & $\begin{array}{l}\text { Ripe fruits are consumed } \\
\text { directly. }\end{array}$ & - \\
\hline 62. & $\begin{array}{l}\text { Jangali } \\
\text { Kanda }\end{array}$ & $\begin{array}{l}\text { Drimia in- } \\
\text { dica (Roxb.) } \\
\text { Jessop }\end{array}$ & $\begin{array}{l}\text { Asparaga- } \\
\text { ceae }\end{array}$ & Herb & $\begin{array}{l}\text { All } \\
\text { season }\end{array}$ & Bulb & Medicinal & - & $\begin{array}{l}\text { Juice of the bulb } \\
\text { is consumed } \\
\text { during piles. }\end{array}$ \\
\hline 63. & $\begin{array}{l}\text { Jungli- } \\
\text { Suran/ } \\
\text { Loth/ } \\
\text { Shevala }\end{array}$ & $\begin{array}{l}\text { Amorpho- } \\
\text { phallus } \\
\text { commutatus } \\
\text { (Schott) } \\
\text { Engl. }\end{array}$ & Araceae & Herb & Monsoon & $\begin{array}{l}\text { Tuber, } \\
\text { Tender } \\
\text { leaves } \\
\text { and Stem }\end{array}$ & $\begin{array}{l}\text { Food } \\
\text { (Sold in } \\
\text { the market } \\
\text { during } \\
\text { season) }\end{array}$ & $\begin{array}{l}\text { 1. Tubers are boiled and } \\
\text { cooked as vegetable. } \\
\text { 2. Tender leaves (Loth) are } \\
\text { steamed and cooked with } \\
\text { onion and garlic. Leaves of } \\
\text { 'Bondar' are added while } \\
\text { cooking to remove the } \\
\text { itchiness. } \\
\text { 3. The leaves are also dried } \\
\text { and stored for off season } \\
\text { consumption. } \\
4 \text {. Tender stem (Shevala) is } \\
\text { cooked as vegetable. }\end{array}$ & - \\
\hline
\end{tabular}




\begin{tabular}{|c|c|c|c|c|c|c|c|c|c|}
\hline $\begin{array}{l}\text { Sr. } \\
\text { No }\end{array}$ & $\begin{array}{l}\text { Local } \\
\text { Name of } \\
\text { Species }\end{array}$ & $\begin{array}{l}\text { Scientific } \\
\text { Name }\end{array}$ & Family & Habit & $\begin{array}{l}\text { Season } \\
\text { of availa- } \\
\text { bility }\end{array}$ & $\begin{array}{l}\text { Plant } \\
\text { part } \\
\text { used }\end{array}$ & $\begin{array}{l}\text { Signifi- } \\
\text { cance }\end{array}$ & $\begin{array}{l}\text { Recipes } \\
\text { (If any) }\end{array}$ & $\begin{array}{l}\text { Medicinal } \\
\text { information }\end{array}$ \\
\hline 64. & Jwari & $\begin{array}{l}\text { Sorghum } \\
\text { bicolor (L.) } \\
\text { Moench }\end{array}$ & Poaceae & Herb & Winter & Seeds & Food & $\begin{array}{l}\text { Powdered seeds are mixed } \\
\text { with water to make bread. }\end{array}$ & - \\
\hline 65. & Kadunimb & $\begin{array}{l}\text { Azadirachta } \\
\text { indicaA. } \\
\text { Juss. }\end{array}$ & Meliaceae & Tree & $\begin{array}{l}\text { All } \\
\text { season }\end{array}$ & Leaves & Medicinal & - & $\begin{array}{l}\text { Leaves decoction } \\
\text { is used for dental } \\
\text { care. }\end{array}$ \\
\hline 66. & $\begin{array}{l}\text { Kadu- } \\
\text { kand/ } \\
\text { Dukkar- } \\
\text { kand }\end{array}$ & $\begin{array}{l}\text { Dioscorea } \\
\text { bulbifera L. }\end{array}$ & $\begin{array}{l}\text { Dioscorea- } \\
\text { ceae }\end{array}$ & Climber & Monsoon & Tuber & Food & $\begin{array}{l}\text { Tuber is kept overnight in } \\
\text { the river so as to dissolve the } \\
\text { bitter taste. It is then boiled } \\
\text { and consumed. }\end{array}$ & - \\
\hline 67. & Kahandal & $\begin{array}{l}\text { Firmiana } \\
\text { simplex (L.) } \\
\text { W.Wight }\end{array}$ & Malvaceae & Tree & Winter & $\begin{array}{l}\text { Seeds and } \\
\text { Gum }\end{array}$ & Food & $\begin{array}{l}\text { 1. Roasted seeds are } \\
\text { consumed. } \\
2 \text {. The gum of the plant is } \\
\text { also consumed by adding it } \\
\text { in various food items. }\end{array}$ & - \\
\hline 68. & Kaharul & $\begin{array}{l}\text { Bauhinia } \\
\text { purpurea L. }\end{array}$ & $\begin{array}{l}\text { Legumino- } \\
\text { sae }\end{array}$ & Tree & Winter & $\begin{array}{l}\text { Young } \\
\text { leaves } \\
\text { and Buds }\end{array}$ & Food & $\begin{array}{l}\text { Young leaves and buds are } \\
\text { consumed as vegetable. }\end{array}$ & - \\
\hline 69. & $\begin{array}{l}\text { Kakad/ } \\
\text { Kakhodshi }\end{array}$ & $\begin{array}{l}\text { Garuga } \\
\text { pinnata } \\
\text { Roxb. }\end{array}$ & Burseraceae & Tree & Summer & Fruits & Food & $\begin{array}{l}\text { Fruits are consumed directly } \\
\text { and also prepared as pickle. }\end{array}$ & - \\
\hline 70. & Kakdi & $\begin{array}{l}\text { Cucumis } \\
\text { satious L. }\end{array}$ & $\begin{array}{l}\text { Cucurbita- } \\
\text { ceae }\end{array}$ & Climber & Summer & Fruits & Food & Direct consumption of fruits & - \\
\hline 71. & Kala kuda & $\begin{array}{l}\text { Wrightia } \\
\text { tinctoria } \\
\text { R.Br. }\end{array}$ & $\begin{array}{l}\text { Apocyna- } \\
\text { ceae }\end{array}$ & Tree & Summer & Flowers & Food & $\begin{array}{l}\text { Flowers are boiled and } \\
\text { cooked in local masalas as } \\
\text { vegetable. }\end{array}$ & - \\
\hline
\end{tabular}




\begin{tabular}{|c|c|c|c|c|c|c|c|c|c|}
\hline $\begin{array}{l}\text { Sr. } \\
\text { No }\end{array}$ & $\begin{array}{l}\text { Local } \\
\text { Name of } \\
\text { Species }\end{array}$ & $\begin{array}{l}\text { Scientific } \\
\text { Name }\end{array}$ & Family & Habit & $\begin{array}{l}\text { Season } \\
\text { of availa- } \\
\text { bility }\end{array}$ & $\begin{array}{l}\text { Plant } \\
\text { part } \\
\text { used }\end{array}$ & $\begin{array}{l}\text { Signifi- } \\
\text { cance }\end{array}$ & $\begin{array}{l}\text { Recipes } \\
\text { (If any) }\end{array}$ & $\begin{array}{l}\text { Medicinal } \\
\text { information }\end{array}$ \\
\hline 72. & $\begin{array}{l}\text { Kali } \\
\text { dhaman }\end{array}$ & $\begin{array}{l}\text { Grewia } \\
\text { tillifolia } \\
\text { Vahl. }\end{array}$ & Malvaceae & Tree & Summer & $\begin{array}{l}\text { Fruits, } \\
\text { Bark and } \\
\text { Roots }\end{array}$ & $\begin{array}{l}\text { Food and } \\
\text { Medicinal }\end{array}$ & Direct consumption of fruits. & $\begin{array}{l}\text { 1. Root extract is } \\
\text { consumed during } \\
\text { stomach ache. } \\
2 \text {. Bark decoction } \\
\text { is consumed } \\
\text { during piles and } \\
\text { bones joints. }\end{array}$ \\
\hline 73. & $\begin{array}{l}\text { Kali } \\
\text { musali }\end{array}$ & $\begin{array}{l}\text { Curculigo } \\
\text { orchiodes } \\
\text { Gaertn. }\end{array}$ & $\begin{array}{l}\text { Hypoxida- } \\
\text { cea }\end{array}$ & Herb & Monsoon & Roots & Medicinal & - & $\begin{array}{l}\text { Root decoction is } \\
\text { useful in treat- } \\
\text { ment of piles and } \\
\text { joint pain. }\end{array}$ \\
\hline 74. & $\begin{array}{l}\text { Kamal } \\
\text { kakadi }\end{array}$ & $\begin{array}{l}\text { Nelumbo } \\
\text { nucifera } \\
\text { Gaertn. }\end{array}$ & $\begin{array}{l}\text { Nelumbo- } \\
\text { naceae }\end{array}$ & Herb & All season & Seeds & Food & Roasted seeds are consumed. & - \\
\hline 75. & Kardai & $\begin{array}{l}\text { Argemone } \\
\text { mexicana L. }\end{array}$ & Asteraceae & Herb & Summer & Seeds & Food & $\begin{array}{l}\text { Seeds are crushed to extract } \\
\text { edible oil which is used in } \\
\text { cooking purposes. }\end{array}$ & - \\
\hline 76. & $\begin{array}{l}\text { Karli/ } \\
\text { Karle/ } \\
\text { Karavli }\end{array}$ & $\begin{array}{l}\text { Momordica } \\
\text { charantia L. }\end{array}$ & $\begin{array}{l}\text { Cucurbita- } \\
\text { ceae }\end{array}$ & Climber & Monsoon & Fruits & Food & $\begin{array}{l}\text { Fruits are boiled \& cooked } \\
\text { as vegetable. }\end{array}$ & - \\
\hline 77. & Kartoli & $\begin{array}{l}\text { Momordica } \\
\text { dioica Roxb. } \\
\text { exWilld. }\end{array}$ & $\begin{array}{l}\text { Cucurbita- } \\
\text { ceae }\end{array}$ & Climber & Monsoon & Fruits & $\begin{array}{l}\text { Food } \\
\text { (Sold in } \\
\text { the market } \\
\text { during } \\
\text { season) }\end{array}$ & $\begin{array}{l}\text { Fruits are boiled \& cooked } \\
\text { in oil as vegetable. }\end{array}$ & - \\
\hline 78. & $\begin{array}{l}\text { Karval/ } \\
\text { Karmal }\end{array}$ & $\begin{array}{l}\text { Dillenia } \\
\text { pentagyna } \\
\text { Roxb. }\end{array}$ & $\begin{array}{l}\text { Dillenia- } \\
\text { ceae }\end{array}$ & Tree & Summer & Flowers & Food & $\begin{array}{l}\text { Flowers are used in } \\
\text { preparation of vegetable. }\end{array}$ & - \\
\hline
\end{tabular}




\begin{tabular}{|c|c|c|c|c|c|c|c|c|c|}
\hline $\begin{array}{l}\text { Sr. } \\
\text { No }\end{array}$ & $\begin{array}{l}\text { Local } \\
\text { Name of } \\
\text { Species }\end{array}$ & $\begin{array}{l}\text { Scientific } \\
\text { Name }\end{array}$ & Family & Habit & $\begin{array}{l}\text { Season } \\
\text { of availa- } \\
\text { bility }\end{array}$ & $\begin{array}{l}\text { Plant } \\
\text { part } \\
\text { used }\end{array}$ & $\begin{array}{l}\text { Signifi- } \\
\text { cance }\end{array}$ & $\begin{array}{l}\text { Recipes } \\
\text { (If any) }\end{array}$ & $\begin{array}{l}\text { Medicinal } \\
\text { information }\end{array}$ \\
\hline 79. & $\begin{array}{l}\text { Karvand/ } \\
\text { Karvanda }\end{array}$ & $\begin{array}{l}\text { Carissa } \\
\text { carandas L. }\end{array}$ & $\begin{array}{l}\text { Apocyna- } \\
\text { ceae }\end{array}$ & Shrub & Summer & Fruits & Food & $\begin{array}{l}\text { 1. Fruits are consumed } \\
\text { directly on ripening. } \\
\text { 2. Raw fruits are also used } \\
\text { in pickles. }\end{array}$ & - \\
\hline 80. & Kate Math & $\begin{array}{l}\text { Amaranthus } \\
\text { spinosus L. }\end{array}$ & $\begin{array}{l}\text { Amaran- } \\
\text { thaceae }\end{array}$ & Herb & Monsoon & Leaves & $\begin{array}{l}\text { Food } \\
\text { (Sold in } \\
\text { the market } \\
\text { during } \\
\text { season) }\end{array}$ & $\begin{array}{l}\text { Leaves are fried and cooked } \\
\text { in oil and masalas as } \\
\text { vegetable. }\end{array}$ & - \\
\hline 81. & Katesawar & $\begin{array}{l}\text { Bombax } \\
\text { ceiba L. }\end{array}$ & Malvaceae & Tree & Summer & Flowers & Food & $\begin{array}{l}\text { Flowers are boiled and } \\
\text { cooked as vegetable. }\end{array}$ & - \\
\hline 82. & Kavadar & $\begin{array}{l}\text { Ensete } \\
\text { superbum } \\
\text { (Roxb.) } \\
\text { Cheesman }\end{array}$ & Musaeae & Herb & Monsoon & $\begin{array}{l}\text { Flowers } \\
\text { and stem }\end{array}$ & Food & $\begin{array}{l}\text { Stem and flower are boiled } \\
\text { and consumed as a vegetable. }\end{array}$ & - \\
\hline 83. & $\begin{array}{l}\text { Kaval- } \\
\text { amba }\end{array}$ & $\begin{array}{l}\text { Trichosan- } \\
\text { thes tricu- } \\
\text { spidata Lour }\end{array}$ & $\begin{array}{l}\text { Cucurbita- } \\
\text { ceae }\end{array}$ & Climber & Monsoon & Roots & Medicinal & - & $\begin{array}{l}\text { Roots decoction } \\
\text { consumed during } \\
\text { urinary infection. }\end{array}$ \\
\hline 84. & $\begin{array}{l}\text { Kavisha/ } \\
\text { Kavisa }\end{array}$ & $\begin{array}{l}\text { Firmiana } \\
\text { colorata } \\
\text { (Roxb.) } \\
\text { R.Br. }\end{array}$ & Malvaceae & Tree & $\begin{array}{l}\text { All } \\
\text { season }\end{array}$ & Bark & Medicinal & - & $\begin{array}{l}\text { Bark decoction is } \\
\text { consumed during } \\
\text { urine infection } \\
\text { and stomach } \\
\text { pain. }\end{array}$ \\
\hline 85. & Kawla & $\begin{array}{l}\text { Smithia } \\
\text { conferta Sm. }\end{array}$ & $\begin{array}{l}\text { Legumino- } \\
\text { sae }\end{array}$ & Herb & Monsoon & Leaves & Food & $\begin{array}{l}\text { Young leaves cooked with } \\
\text { spices as vegetable. }\end{array}$ & - \\
\hline 86. & Keni & $\begin{array}{l}\text { Commelina } \\
\text { benghalensis } \\
\text { L. }\end{array}$ & $\begin{array}{l}\text { Commeli- } \\
\text { naceae }\end{array}$ & Herb & Monsoon & Leaves & Food & $\begin{array}{l}\text { Young leaves are boiled and } \\
\text { cooked in oil as vegetable. }\end{array}$ & - \\
\hline
\end{tabular}




\begin{tabular}{|c|c|c|c|c|c|c|c|c|c|}
\hline $\begin{array}{l}\text { Sr. } \\
\text { No }\end{array}$ & $\begin{array}{l}\text { Local } \\
\text { Name of } \\
\text { Species }\end{array}$ & $\begin{array}{l}\text { Scientific } \\
\text { Name }\end{array}$ & Family & Habit & $\begin{array}{l}\text { Season } \\
\text { of availa- } \\
\text { bility }\end{array}$ & $\begin{array}{l}\text { Plant } \\
\text { part } \\
\text { used }\end{array}$ & $\begin{array}{l}\text { Signifi- } \\
\text { cance }\end{array}$ & $\begin{array}{l}\text { Recipes } \\
\text { (If any) }\end{array}$ & $\begin{array}{l}\text { Medicinal } \\
\text { information }\end{array}$ \\
\hline 87. & $\begin{array}{l}\text { Khadak- } \\
\text { tera/ } \\
\text { Teralu }\end{array}$ & $\begin{array}{l}\text { Ariopsis } \\
\text { peltata } \\
\text { Nimmo }\end{array}$ & Araceae & Herb & Monsoon & Leaves & Food & $\begin{array}{l}\text { 1. Leaves are cooked as } \\
\text { vegetable with oil and onion. } \\
2 \text {. Leaves are also rolled to } \\
\text { make spirals along with } \\
\text { chana paste which is con- } \\
\text { sumed after shallow frying. } \\
\text { 3. Leaves are directly added } \\
\text { to curry as garnish. }\end{array}$ & - \\
\hline 88. & $\begin{array}{l}\text { Khajur/ } \\
\text { Shindi }\end{array}$ & $\begin{array}{l}\text { Phoenix } \\
\text { sylvestrus } \\
\text { (L.) Roxb. }\end{array}$ & Arecaceae & Tree & Summer & Fruits and & $\begin{array}{l}\text { Food } \\
\text { Bark sap }\end{array}$ & $\begin{array}{l}\text { Fruits are consumed directly } \\
\text { while the sap is consumed } \\
\text { as 'Madi/Neera' } \\
\text { (Local alcohol). }\end{array}$ & - \\
\hline 89. & $\begin{array}{l}\text { Kharbat/ } \\
\text { Kharbati }\end{array}$ & $\begin{array}{l}\text { Grewia } \\
\text { abutilifolia } \\
\text { Vent. ex Juss. }\end{array}$ & Malvaceae & Shrub & $\begin{array}{l}\text { All } \\
\text { season }\end{array}$ & Fruits & Food & Direct consumption of fruits. & - \\
\hline 90. & Kharsheng & $\begin{array}{l}\text { Raderma- } \\
\text { chera xylo- } \\
\text { carpa(Roxb.) } \\
\text { Roxb. ex K. } \\
\text { Schum. }\end{array}$ & $\begin{array}{l}\text { Bignonia- } \\
\text { ceae }\end{array}$ & Tree & Monsoon & Fruits & Food & $\begin{array}{l}\text { Young pods are boiled to } \\
\text { remove bitterness and } \\
\text { cooked as vegetable. }\end{array}$ & - \\
\hline 91. & $\begin{array}{l}\text { Khura- } \\
\text { sani/ } \\
\text { Khurasni }\end{array}$ & $\begin{array}{l}\text { Guizotia } \\
\text { abyssinica } \\
\text { (L.f.) Cass. }\end{array}$ & Compositae & Herb & Summer & $\begin{array}{l}\text { Seeds and } \\
\text { Leaves }\end{array}$ & Food & $\begin{array}{l}\text { 1. Oil is extracted from seeds. } \\
\text { 2. Tender leaves steamed } \\
\text { and cooked with local } \\
\text { masalas as vegetable. }\end{array}$ & - \\
\hline 92. & Koprya & $\begin{array}{l}\text { Alternan- } \\
\text { thera } \\
\text { sessilis(L.) } \\
\text { R.Br. ex DC. }\end{array}$ & $\begin{array}{l}\text { Amaran- } \\
\text { thaceae }\end{array}$ & Herb & Monsoon & Leaves & Food & $\begin{array}{l}\text { Leaves are boiled and } \\
\text { cooked as vegetable. }\end{array}$ & - \\
\hline 93. & Vazkand & $\begin{array}{l}\text { Dioscorea } \\
\text { hispida } \\
\text { Dennst. }\end{array}$ & $\begin{array}{l}\text { Dioscorea- } \\
\text { ceae }\end{array}$ & Climber & Monsoon & Tuber & Food & $\begin{array}{l}\text { Tubers are boiled and } \\
\text { cooked as vegetable. }\end{array}$ & - \\
\hline
\end{tabular}




\begin{tabular}{|c|c|c|c|c|c|c|c|c|c|}
\hline $\begin{array}{l}\text { Sr. } \\
\text { No }\end{array}$ & $\begin{array}{l}\text { Local } \\
\text { Name of } \\
\text { Species }\end{array}$ & $\begin{array}{l}\text { Scientific } \\
\text { Name }\end{array}$ & Family & Habit & $\begin{array}{l}\text { Season } \\
\text { of availa- } \\
\text { bility }\end{array}$ & $\begin{array}{l}\text { Plant } \\
\text { part } \\
\text { used }\end{array}$ & $\begin{array}{l}\text { Signifi- } \\
\text { cance }\end{array}$ & $\begin{array}{l}\text { Recipes } \\
\text { (If any) }\end{array}$ & $\begin{array}{l}\text { Medicinal } \\
\text { information }\end{array}$ \\
\hline 94. & $\begin{array}{l}\text { Koshimb/ } \\
\text { Koshim/ } \\
\text { Kusum }\end{array}$ & $\begin{array}{l}\text { Schleichera } \\
\text { oleosa (Lour.) } \\
\text { Oken. }\end{array}$ & $\begin{array}{l}\text { Sapinda- } \\
\text { ceae }\end{array}$ & Tree & Winter & $\begin{array}{l}\text { Leaves } \\
\text { and Seeds }\end{array}$ & Medicinal & Seed oil is used in vegetable. & $\begin{array}{l}\text { Leaves decoction } \\
\text { given for } \\
\text { vomiting. }\end{array}$ \\
\hline 95. & $\begin{array}{l}\text { Kotham- } \\
\text { bal/ } \\
\text { Kothimbir }\end{array}$ & $\begin{array}{l}\text { Coriandrum } \\
\text { sativum L. }\end{array}$ & Apiaceae & Herb & $\begin{array}{l}\text { All } \\
\text { season }\end{array}$ & Leaves & Food & $\begin{array}{l}\text { Leaves cooked as vegetable } \\
\text { or consumed directly by add- } \\
\text { ing in the curry or as garnish. }\end{array}$ & \\
\hline 96. & $\begin{array}{l}\text { Kudie/ } \\
\text { Kuda/ } \\
\text { Kod/ } \\
\text { Kudva }\end{array}$ & $\begin{array}{l}\text { Holarrhena } \\
\text { pubescens } \\
\text { Wall. ex } \\
\text { G.Don }\end{array}$ & $\begin{array}{l}\text { Apocyna- } \\
\text { ceae }\end{array}$ & Shrub & Winter & Flowers & Food & $\begin{array}{l}\text { Flowers are boiled and } \\
\text { cooked as vegetable. }\end{array}$ & - \\
\hline 97. & $\begin{array}{l}\text { Kuharool// } \\
\text { Shid }\end{array}$ & $\begin{array}{l}\text { Bauhinia } \\
\text { racemosa } \\
\text { Lam. }\end{array}$ & $\begin{array}{l}\text { Legumino- } \\
\text { sae }\end{array}$ & Tree & Winter & Leaves & Food & $\begin{array}{l}\text { Leaves are boiled and } \\
\text { cooked as vegetable. }\end{array}$ & - \\
\hline 98. & $\begin{array}{l}\text { Kuli/ } \\
\text { Kovali- } \\
\text { bhaji }\end{array}$ & $\begin{array}{l}\text { Chloro- } \\
\text { phytum } \\
\text { tuberosum } \\
\text { (Roxb.) } \\
\text { Baker }\end{array}$ & $\begin{array}{l}\text { Asparaga- } \\
\text { ceae }\end{array}$ & Herb & Monsoon & $\begin{array}{l}\text { Young } \\
\text { leaves }\end{array}$ & Food & $\begin{array}{l}\text { Leaves are boiled and } \\
\text { cooked as vegetable. }\end{array}$ & - \\
\hline 99. & Kumbha & $\begin{array}{l}\text { Careya } \\
\text { arborea } \\
\text { Roxb. }\end{array}$ & $\begin{array}{l}\text { Lecythida- } \\
\text { ceae }\end{array}$ & Tree & $\begin{array}{l}\text { All } \\
\text { season }\end{array}$ & Bark & Medicinal & - & $\begin{array}{l}\text { Bark powder is } \\
\text { consumed during } \\
\text { stomach pain }\end{array}$ \\
\hline 100. & Kurdu & $\begin{array}{l}\text { Celosia } \\
\text { argentea } \mathrm{L} .\end{array}$ & $\begin{array}{l}\text { Amaran- } \\
\text { thaceae }\end{array}$ & Herb & Monsoon & Leaves & Food & $\begin{array}{l}\text { Young leaves are steamed } \\
\text { and cooked with onion and } \\
\text { local masalas as vegetable. }\end{array}$ & - \\
\hline 101. & Kusar & $\begin{array}{l}\text { Jasminum } \\
\text { malabaricum } \\
\text { Wight }\end{array}$ & Oleaceae & Climber & Monsoon & Fruits & Food & Fruits are consumed directly. & - \\
\hline
\end{tabular}




\begin{tabular}{|c|c|c|c|c|c|c|c|c|c|}
\hline $\begin{array}{l}\text { Sr. } \\
\text { No }\end{array}$ & $\begin{array}{l}\text { Local } \\
\text { Name of } \\
\text { Species }\end{array}$ & $\begin{array}{l}\text { Scientific } \\
\text { Name }\end{array}$ & Family & Habit & $\begin{array}{l}\text { Season } \\
\text { of availa- } \\
\text { bility }\end{array}$ & $\begin{array}{l}\text { Plant } \\
\text { part } \\
\text { used }\end{array}$ & $\begin{array}{l}\text { Signifi- } \\
\text { cance }\end{array}$ & $\begin{array}{l}\text { Recipes } \\
\text { (If any) }\end{array}$ & $\begin{array}{l}\text { Medicinal } \\
\text { information }\end{array}$ \\
\hline 102. & Lokhandi & $\begin{array}{l}\text { Ixora } \\
\text { brachiata } \\
\text { Roxb. }\end{array}$ & Rubiaceae & Tree & $\begin{array}{l}\text { All } \\
\text { season }\end{array}$ & Leaves & Medicinal & - & $\begin{array}{l}\text { Leaf powder is } \\
\text { consumed during } \\
\text { cough. }\end{array}$ \\
\hline 103. & Lundha & $\begin{array}{l}\text { Dioscorea } \\
\text { pentaphylla } \\
\text { var. } \\
\text { jacque- } \\
\text { montii } \\
\text { (Hook.f.) } \\
\text { Prain \& } \\
\text { Burkill }\end{array}$ & $\begin{array}{l}\text { Dioscorea- } \\
\text { ceae }\end{array}$ & Climber & Monsoon & $\begin{array}{l}\text { Young } \\
\text { shoots } \\
\text { and } \\
\text { tubers }\end{array}$ & Food & $\begin{array}{l}\text { 1. Tubers are boiled and } \\
\text { cooked as vegetable. } \\
\text { 2. Young shoots are also } \\
\text { boiled and cooked as } \\
\text { vegetable. }\end{array}$ & - \\
\hline 104. & Maswadi & $\begin{array}{l}\text { Ocimum } \\
\text { sps }\end{array}$ & Lamiaceae & Herb & $\begin{array}{l}\text { All } \\
\text { season }\end{array}$ & Leaves & Food & $\begin{array}{l}\text { Leaves are used in local } \\
\text { sauce preparation }\end{array}$ & - \\
\hline 105. & $\begin{array}{l}\text { Math } \\
\text { bhaji/ } \\
\text { Getna }\end{array}$ & $\begin{array}{l}\text { Digera } \\
\text { muricata (L.) } \\
\text { Mart. }\end{array}$ & $\begin{array}{l}\text { Amaran- } \\
\text { thaceae }\end{array}$ & Herb & Monsoon & Leaves & Food & $\begin{array}{l}\text { Young leaves are steamed } \\
\text { and stir fried with onion, } \\
\text { garlic and chili to make the } \\
\text { vegetable. }\end{array}$ & - \\
\hline 106. & Mayalu & $\begin{array}{l}\text { Basella } \\
\text { alba L. }\end{array}$ & Basellaceae & Climber & Monsoon & Leaves & Food & $\begin{array}{l}\text { Leaves are specially used to } \\
\text { make Fritters. It is also con- } \\
\text { sumed as a vegetable or } \\
\text { added in curry preparation. }\end{array}$ & - \\
\hline 107. & Meki & $\begin{array}{l}\text { Cucumis } \\
\text { setosus } \\
\text { Cogn. }\end{array}$ & $\begin{array}{l}\text { Cucurbita- } \\
\text { ceae }\end{array}$ & Climber & Monsoon & Fruits & Food & $\begin{array}{l}\text { Raw fruits are consumed } \\
\text { directly. }\end{array}$ & - \\
\hline 108. & $\begin{array}{l}\text { Moha/ } \\
\text { Moh/ } \\
\text { Mohati }\end{array}$ & \begin{tabular}{l|} 
Madhuca \\
longifolia \\
var. latifolia \\
(Roxb.) \\
A.Chev.
\end{tabular} & Sapotaceae & Tree & Summer & $\begin{array}{l}\text { Seeds and } \\
\text { flowers }\end{array}$ & Food & $\begin{array}{l}\text { 1. Flowers are cooked and } \\
\text { consumed as vegetable. } \\
2 \text {. Seeds are crushed for oil } \\
\text { which is used during } \\
\text { cooking. }\end{array}$ & - \\
\hline
\end{tabular}




\begin{tabular}{|c|c|c|c|c|c|c|c|c|c|}
\hline $\begin{array}{l}\text { Sr. } \\
\text { No }\end{array}$ & $\begin{array}{l}\text { Local } \\
\text { Name of } \\
\text { Species }\end{array}$ & $\begin{array}{l}\text { Scientific } \\
\text { Name }\end{array}$ & Family & Habit & $\begin{array}{l}\text { Season } \\
\text { of availa- } \\
\text { bility }\end{array}$ & $\begin{array}{l}\text { Plant } \\
\text { part } \\
\text { used }\end{array}$ & $\begin{array}{l}\text { Signifi- } \\
\text { cance }\end{array}$ & $\begin{array}{l}\text { Recipes } \\
\text { (If any) }\end{array}$ & $\begin{array}{l}\text { Medicinal } \\
\text { information }\end{array}$ \\
\hline 109. & $\begin{array}{l}\text { Mokha/ } \\
\text { Mukh- } \\
\text { pala/ } \\
\text { Moka }\end{array}$ & $\begin{array}{l}\text { Schrebera } \\
\text { swietenioides } \\
\text { Roxb. }\end{array}$ & Oleaceae & Tree & $\begin{array}{l}\text { Late } \\
\text { Summer }\end{array}$ & $\begin{array}{l}\text { Fruits and } \\
\text { Leaves }\end{array}$ & Food & $\begin{array}{l}\text { Young leaves and fruits are } \\
\text { boiled and cooked as } \\
\text { vegetable. }\end{array}$ & - \\
\hline 110. & Morvel & $\begin{array}{l}\text { Clematis } \\
\text { gouriana } \\
\text { Roxb.ex DC. }\end{array}$ & $\begin{array}{l}\text { Ranuncula- } \\
\text { ceae }\end{array}$ & Climber & Monsoon & Fruits & $\begin{array}{l}\text { Food and } \\
\text { Medicinal }\end{array}$ & $\begin{array}{l}\text { Leaves boiled and cooked } \\
\text { with spices }\end{array}$ & $\begin{array}{l}\text { Fruit extract used } \\
\text { in ear ache }\end{array}$ \\
\hline 111. & $\begin{array}{l}\text { Murud } \\
\text { sheng/ } \\
\text { Aati }\end{array}$ & $\begin{array}{l}\text { Helicteres } \\
\text { isora } \mathrm{L} .\end{array}$ & Malvaceae & Shrub & Summer & Pods & Medicinal & - & $\begin{array}{l}\text { Fruit decoction is } \\
\text { used for stomach } \\
\text { pain. } \\
\text { 1. Crushed } \\
\text { powder is } \\
\text { consumed with } \\
\text { water on scor- } \\
\text { pion bite. }\end{array}$ \\
\hline 112. & $\begin{array}{l}\text { Nachani/ } \\
\text { Nagli }\end{array}$ & \begin{tabular}{l|} 
Eleusine \\
coracana $(\mathrm{L})$. \\
Gaertn.
\end{tabular} & Poaceae & Herb & Monsoon & Flowers & Food & $\begin{array}{l}\text { Grain powder is mixed with } \\
\text { water and used for making } \\
\text { 'bread'. }\end{array}$ & - \\
\hline 113. & Nadukali & $\begin{array}{l}\text { Pavetta } \\
\text { indica L. }\end{array}$ & Rubiaceae & Shrub & Summer & Flowers & Food & $\begin{array}{l}\text { Flowers are boiled and } \\
\text { cooked as vegetable. }\end{array}$ & - \\
\hline 114. & Nalbhaji & $\begin{array}{l}\text { Ipomoea } \\
\text { aquatica } \\
\text { Forssk. }\end{array}$ & $\begin{array}{l}\text { Convol- } \\
\text { vulaceae }\end{array}$ & Climber & Winter & $\begin{array}{l}\text { Leaves } \\
\text { and Stem }\end{array}$ & Food & $\begin{array}{l}\text { Leaves and stem are cooked } \\
\text { with onion and local masala } \\
\text { as vegetable. }\end{array}$ & - \\
\hline 115. & Nilgiri & $\begin{array}{l}\text { Eucalyptus } \\
\text { globulus } \\
\text { Labill. }\end{array}$ & Myrtaceae & Tree & $\begin{array}{l}\text { All } \\
\text { season }\end{array}$ & Leaves & Medicinal & - & $\begin{array}{l}\text { Leaf extract used } \\
\text { on dental } \\
\text { problem and also } \\
\text { used in common } \\
\text { cold and cough. }\end{array}$ \\
\hline 116. & Nirgudi & $\begin{array}{l}\text { Vitex } \\
\text { negundo L. }\end{array}$ & Lamiaceae & Shrub & $\begin{array}{l}\text { All } \\
\text { season }\end{array}$ & Leaves & Medicinal & - & $\begin{array}{l}\text { Leaf extract used } \\
\text { for ear pain. }\end{array}$ \\
\hline
\end{tabular}




\begin{tabular}{|c|c|c|c|c|c|c|c|c|c|}
\hline $\begin{array}{l}\text { Sr. } \\
\text { No }\end{array}$ & $\begin{array}{l}\text { Local } \\
\text { Name of } \\
\text { Species }\end{array}$ & $\begin{array}{l}\text { Scientific } \\
\text { Name }\end{array}$ & Family & Habit & $\begin{array}{l}\text { Season } \\
\text { of availa- } \\
\text { bility }\end{array}$ & $\begin{array}{l}\text { Plant } \\
\text { part } \\
\text { used }\end{array}$ & $\begin{array}{l}\text { Signifi- } \\
\text { cance }\end{array}$ & $\begin{array}{l}\text { Recipes } \\
\text { (If any) }\end{array}$ & $\begin{array}{l}\text { Medicinal } \\
\text { information }\end{array}$ \\
\hline 117. & Ova & $\begin{array}{l}\text { Plectra- } \\
\text { nthusam- } \\
\text { boinicus } \\
\text { (Lour.) } \\
\text { Spreng. }\end{array}$ & Lamiaceae & Herb & $\begin{array}{l}\text { All } \\
\text { season }\end{array}$ & Leaves & Food & $\begin{array}{l}\text { Leaves used to make Fritters } \\
\text { and also added in curries } \\
\text { for taste. }\end{array}$ & - \\
\hline 118. & Palas & $\begin{array}{l}\text { Butea } \\
\text { monosperma } \\
\text { (Lam.) } \\
\text { Taub. }\end{array}$ & $\begin{array}{l}\text { Legumino- } \\
\text { sae }\end{array}$ & Tree & Winter & Flowers & $\begin{array}{l}\text { Food and } \\
\text { Medicinal }\end{array}$ & $\begin{array}{l}\text { Flowers are used to make } \\
\text { vegetable. }\end{array}$ & $\begin{array}{l}\text { Branch decoction } \\
\text { is consumed } \\
\text { during sore } \\
\text { throat. }\end{array}$ \\
\hline 119. & Papai & $\begin{array}{l}\text { Carica } \\
\text { papaya } \mathrm{L} .\end{array}$ & Caricaceae & Tree & $\begin{array}{l}\text { All } \\
\text { season }\end{array}$ & Fruit & $\begin{array}{l}\text { Food and } \\
\text { Medicinal }\end{array}$ & $\begin{array}{l}\text { Fruits are consumed directly } \\
\text { on ripening. }\end{array}$ & $\begin{array}{l}\text { Milky latex is } \\
\text { consumed during } \\
\text { dental problem. }\end{array}$ \\
\hline 120. & Papdi & $\begin{array}{l}\text { Holoptelea } \\
\text { integrifolia } \\
\text { Planch. }\end{array}$ & Ulmaceae & Tree & Summer & Seeds & $\begin{array}{l}\text { Food and } \\
\text { Medicinal }\end{array}$ & Seeds consumed directly & $\begin{array}{l}\text { Bark extract is } \\
\text { used for dental } \\
\text { diseases. }\end{array}$ \\
\hline 121. & $\begin{array}{l}\text { Raan- } \\
\text { Pavata }\end{array}$ & $\begin{array}{l}\text { Lablab } \\
\text { purpureus } \\
\text { (L.) Sweet }\end{array}$ & $\begin{array}{l}\text { Legumino- } \\
\text { sae }\end{array}$ & Climber & Winter & Seeds & Food & $\begin{array}{l}\text { Seeds are cooked as } \\
\text { vegetable. }\end{array}$ & - \\
\hline 122. & Pave & $\begin{array}{l}\text { Cheiloco- } \\
\text { stusspeciosus } \\
\text { (J.Koenig) } \\
\text { C.D.Spech }\end{array}$ & Costaceae & Herb & Monsoon & $\begin{array}{l}\text { Leaves } \\
\text { and Stem }\end{array}$ & Food & $\begin{array}{l}\text { Tender leaves and stem are } \\
\text { added to curry directly. }\end{array}$ & - \\
\hline 123. & $\begin{array}{l}\text { Pendara/ } \\
\text { Pendhar }\end{array}$ & $\begin{array}{l}\text { Tamilnadia } \\
\text { uliginosa } \\
\text { (Retz.) } \\
\text { Tirveng. \& } \\
\text { Sastre }\end{array}$ & Rubiaceae & Tree & Winter & Fruits & $\begin{array}{l}\text { Food } \\
\text { (Sold in } \\
\text { the market } \\
\text { during } \\
\text { season) }\end{array}$ & $\begin{array}{l}\text { Young fruits are boiled and } \\
\text { cooked for consumption. }\end{array}$ & - \\
\hline
\end{tabular}




\begin{tabular}{|c|c|c|c|c|c|c|c|c|c|}
\hline $\begin{array}{l}\text { Sr. } \\
\text { No }\end{array}$ & $\begin{array}{l}\text { Local } \\
\text { Name of } \\
\text { Species }\end{array}$ & $\begin{array}{l}\text { Scientific } \\
\text { Name }\end{array}$ & Family & Habit & $\begin{array}{l}\text { Season } \\
\text { of availa- } \\
\text { bility }\end{array}$ & $\begin{array}{l}\text { Plant } \\
\text { part } \\
\text { used }\end{array}$ & $\begin{array}{l}\text { Signifi- } \\
\text { cance }\end{array}$ & $\begin{array}{l}\text { Recipes } \\
\text { (If any) }\end{array}$ & $\begin{array}{l}\text { Medicinal } \\
\text { information }\end{array}$ \\
\hline 124. & Pimpal & $\begin{array}{l}\text { Ficus } \\
\text { religiosa L. }\end{array}$ & Moraceae & Tree & $\begin{array}{l}\text { All } \\
\text { season }\end{array}$ & Bark & Medicinal & - & $\begin{array}{l}\text { Bark powder is } \\
\text { mixed with water } \\
\text { and consumed } \\
\text { during stomach } \\
\text { pain. }\end{array}$ \\
\hline 125. & $\begin{array}{l}\text { Ran } \\
\text { shegut }\end{array}$ & $\begin{array}{l}\text { Moringa } \\
\text { concanensis } \\
\text { Nimmo }\end{array}$ & $\begin{array}{l}\text { Moringa- } \\
\text { ceae }\end{array}$ & Tree & $\begin{array}{l}\text { All } \\
\text { season }\end{array}$ & $\begin{array}{l}\text { Bark and } \\
\text { Pods }\end{array}$ & $\begin{array}{l}\text { Food and } \\
\text { Medicinal }\end{array}$ & $\begin{array}{l}\text { Pods are boiled and cooked } \\
\text { with local masala as } \\
\text { vegetable. }\end{array}$ & $\begin{array}{l}\text { Bark decoction is } \\
\text { consumed during } \\
\text { worm infection. }\end{array}$ \\
\hline 126. & Ran til & $\begin{array}{l}\text { Sesamumi } \\
\text { ndicum L. }\end{array}$ & Pedaliaceae & Herb & Monsoon & Seeds & Food & $\begin{array}{l}\text { The seeds of the plants are } \\
\text { crushed to extract edible oil. }\end{array}$ & - \\
\hline 127. & Ranvanga & $\begin{array}{l}\text { Solanum } \\
\text { anguivi } \\
\text { Lam. }\end{array}$ & Solanaceae & Shrub & Winter & Fruits & Food & $\begin{array}{l}\text { Fruits are cooked and stir } \\
\text { fried with onion, garlic and } \\
\text { chilly. }\end{array}$ & - \\
\hline 128. & $\begin{array}{l}\text { Ratalu/ } \\
\text { Ratala }\end{array}$ & $\begin{array}{l}\text { Ipomoea } \\
\text { batatas (L.) } \\
\text { Lam. }\end{array}$ & $\begin{array}{l}\text { Convolvu- } \\
\text { laceae }\end{array}$ & Climber & $\begin{array}{l}\text { All } \\
\text { season }\end{array}$ & Tuber & $\begin{array}{l}\text { Food } \\
\text { (Sold in } \\
\text { the market } \\
\text { during } \\
\text { season) }\end{array}$ & $\begin{array}{l}\text { Tubers are boiled and } \\
\text { cooked as vegetable. }\end{array}$ & - \\
\hline 129. & $\begin{array}{l}\text { Ringani/ } \\
\text { Ringni }\end{array}$ & $\begin{array}{l}\text { Solanum } \\
\text { xantho- } \\
\text { carpum } \\
\text { Schrad. \& } \\
\text { H. Wendl }\end{array}$ & Solanaceae & Herb & Monsoon & Fruits & Food & $\begin{array}{l}\text { Fruits are cooked and } \\
\text { consumed as vegetable }\end{array}$ & - \\
\hline 130 . & $\begin{array}{l}\text { Sabar/ } \\
\text { Sabarkand }\end{array}$ & $\begin{array}{l}\text { Euphorbia } \\
\text { nerifolia L. }\end{array}$ & $\begin{array}{l}\text { Euphorbia- } \\
\text { ceae }\end{array}$ & Shrub & $\begin{array}{l}\text { All } \\
\text { season }\end{array}$ & Stem & Medicinal & - & $\begin{array}{l}\text { Stem decoction is } \\
\text { consumed during } \\
\text { cough. }\end{array}$ \\
\hline 131. & $\begin{array}{l}\text { Sadhadi/ } \\
\text { Aine }\end{array}$ & $\begin{array}{l}\text { Terminalia } \\
\text { tomentosa } \\
\text { Wight } \\
\text { \& Arn. }\end{array}$ & $\begin{array}{l}\text { Combreta- } \\
\text { ceae }\end{array}$ & Tree & Summer & Fruits & Food & Young fruits are edible. & - \\
\hline
\end{tabular}




\begin{tabular}{|c|c|c|c|c|c|c|c|c|c|}
\hline $\begin{array}{l}\text { Sr. } \\
\text { No }\end{array}$ & $\begin{array}{l}\text { Local } \\
\text { Name of } \\
\text { Species }\end{array}$ & $\begin{array}{l}\text { Scientific } \\
\text { Name }\end{array}$ & Family & Habit & $\begin{array}{l}\text { Season } \\
\text { of availa- } \\
\text { bility }\end{array}$ & $\begin{array}{l}\text { Plant } \\
\text { part } \\
\text { used }\end{array}$ & $\begin{array}{l}\text { Signifi- } \\
\text { cance }\end{array}$ & $\begin{array}{l}\text { Recipes } \\
\text { (If any) }\end{array}$ & $\begin{array}{l}\text { Medicinal } \\
\text { information }\end{array}$ \\
\hline 132. & Safedkuda & $\begin{array}{l}\text { Holarrhena } \\
\text { antidysen- } \\
\text { terica(Roth) } \\
\text { Wall. ex } \\
\text { A.DC. }\end{array}$ & $\begin{array}{l}\text { Apocyna- } \\
\text { ceae }\end{array}$ & Tree & Summer & Flowers & Food & $\begin{array}{l}\text { Flower are boiled and } \\
\text { cooked as vegetable. }\end{array}$ & - \\
\hline 133. & Sag & $\begin{array}{l}\text { Tectona } \\
\text { grandis L.f. }\end{array}$ & Lamiaceae & Tree & $\begin{array}{l}\text { All } \\
\text { season }\end{array}$ & Seeds & Medicinal & - & $\begin{array}{l}\text { Powder of seeds } \\
\text { is mixed with } \\
\text { water and } \\
\text { consumed for } \\
\text { kidney stone and } \\
\text { Renal problems. }\end{array}$ \\
\hline 134. & $\begin{array}{l}\text { Sapud/ } \\
\text { Dinda/ } \\
\text { Dini }\end{array}$ & $\begin{array}{l}\text { Leea } \\
\text { macrophylla } \\
\text { Roxb. ex } \\
\text { Hornem. }\end{array}$ & Vitaceae & Shrub & Winter & Leaves & Food & $\begin{array}{l}\text { Tender leaves are cooked } \\
\text { with spices as vegetable. }\end{array}$ & - \\
\hline 135. & Sarambal & $\begin{array}{l}\text { Justicia } \\
\text { procumbens } \\
\text { L. }\end{array}$ & $\begin{array}{l}\text { Acantha- } \\
\text { ceae }\end{array}$ & Herb & Monsoon & Leaves & Food & $\begin{array}{l}\text { Young leaves boiled and } \\
\text { cooked as vegetable. }\end{array}$ & - \\
\hline 136. & $\begin{array}{l}\text { Shevaga/ } \\
\text { Shevga/ } \\
\text { Sagava/ } \\
\text { Shegalu }\end{array}$ & $\begin{array}{l}\text { Moringa } \\
\text { oleifera } \\
\text { Lam. }\end{array}$ & $\begin{array}{l}\text { Moringa- } \\
\text { ceae }\end{array}$ & Tree & $\begin{array}{l}\text { All } \\
\text { season }\end{array}$ & $\begin{array}{l}\text { Flowers, } \\
\text { Fruits and } \\
\text { Leaves }\end{array}$ & Food & $\begin{array}{l}\text { Young leaves, fruits and } \\
\text { flowers are cooked and } \\
\text { consumed as vegetable. }\end{array}$ & - \\
\hline 137. & $\begin{array}{l}\text { Siral/ } \\
\text { Dodka }\end{array}$ & $\begin{array}{l}\text { Luffa } \\
\text { acutangula } \\
\text { (L.) Roxb. }\end{array}$ & $\begin{array}{l}\text { Cucurbita- } \\
\text { ceae }\end{array}$ & Climber & Monsoon & Fruit & Food & Fruit cooked as vegetable. & - \\
\hline 138. & Sol & $\begin{array}{l}\begin{array}{l}\text { Cryptoco- } \\
\text { ryne spiralis } \\
\text { (Retz.) }\end{array} \\
\text { Fisch. ex } \\
\text { Wydler }\end{array}$ & Araceae & Herb & Monsoon & $\begin{array}{l}\text { Young } \\
\text { leaves }\end{array}$ & Food & $\begin{array}{l}\text { Leaves are mixed with Gram } \\
\text { flour to make Fritters and } \\
\text { then consumed. }\end{array}$ & - \\
\hline
\end{tabular}




\begin{tabular}{|c|c|c|c|c|c|c|c|c|c|}
\hline $\begin{array}{l}\text { Sr. } \\
\text { No }\end{array}$ & $\begin{array}{l}\text { Local } \\
\text { Name of } \\
\text { Species }\end{array}$ & $\begin{array}{l}\text { Scientific } \\
\text { Name }\end{array}$ & Family & Habit & $\begin{array}{l}\text { Season } \\
\text { of availa- } \\
\text { bility }\end{array}$ & $\begin{array}{l}\text { Plant } \\
\text { part } \\
\text { used }\end{array}$ & $\begin{array}{l}\text { Signifi- } \\
\text { cance }\end{array}$ & $\begin{array}{l}\text { Recipes } \\
\text { (If any) }\end{array}$ & $\begin{array}{l}\text { Medicinal } \\
\text { information }\end{array}$ \\
\hline 139. & Supali & $\begin{array}{l}\text { Cleome } \\
\text { viscosa } \mathrm{L} .\end{array}$ & Cleomaceae & Herb & Monsoon & Pod & Medicinal & $\begin{array}{l}\text { Pods are boiled and cooked } \\
\text { as vegetable. }\end{array}$ & - \\
\hline 140 . & Suran & $\begin{array}{l}\text { Amorpho- } \\
\text { phallus } \\
\text { paeoniifolius } \\
\text { (Dennst.) } \\
\text { Nicolson }\end{array}$ & Araceae & Herb & Monsoon & Tubers & Food & $\begin{array}{l}\text { Tubers are boiled and } \\
\text { cooked with local masala } \\
\text { as vegetable. }\end{array}$ & - \\
\hline 141. & Tadgole & $\begin{array}{l}\text { Borrassus } \\
\text { flabellifer L. }\end{array}$ & Arecaceae & Tree & Summer & Fruits & Food & Fruits are consumed directly. & - \\
\hline 142. & $\begin{array}{l}\text { Tagadi/ } \\
\text { Tagda/ } \\
\text { Taag }\end{array}$ & $\begin{array}{l}\text { Crotalaria } \\
\text { juncea L. }\end{array}$ & $\begin{array}{l}\text { Legumino- } \\
\text { sae }\end{array}$ & Shrub & Monsoon & Flowers & Food & $\begin{array}{l}\text { Flowers are boiled and } \\
\text { cooked as vegetable. }\end{array}$ & - \\
\hline 143. & $\begin{array}{l}\text { Tambat/ } \\
\text { Atrun/ } \\
\text { Gugarval }\end{array}$ & $\begin{array}{l}\text { Flacourtia } \\
\text { indica } \\
\text { (Burm.f.) } \\
\text { Merr. }\end{array}$ & Salicaceae & Tree & Monsoon & Fruits & Food & Fruits are consumed directly. & - \\
\hline 144. & Tandala & $\begin{array}{l}\text { Amaranthus } \\
\text { tricolor L. }\end{array}$ & $\begin{array}{l}\text { Amaran- } \\
\text { thaceae }\end{array}$ & Herb & Monsoon & Leaves & Food & $\begin{array}{l}\text { Leaves are boiled and } \\
\text { cooked as vegetable. }\end{array}$ & - \\
\hline 145. & Tantani & $\begin{array}{l}\text { Lantana } \\
\text { camara L. }\end{array}$ & $\begin{array}{l}\text { Verbena- } \\
\text { ceae }\end{array}$ & Shrub & Winter & Fruits & Food & Fruits are consumed directly. & - \\
\hline 146. & Tanvel & \begin{tabular}{l|} 
Cocculus \\
hirsutus(L.) \\
W.Theob.
\end{tabular} & $\begin{array}{l}\text { Menisper- } \\
\text { maceae }\end{array}$ & Climber & Monsoon & Leaves & Food & $\begin{array}{l}\text { Leaves boiled and cooked in } \\
\text { oil as vegetable. }\end{array}$ & - \\
\hline 147. & $\begin{array}{l}\text { Taravata/ } \\
\text { Tarota/ } \\
\text { Takhata/ } \\
\text { Atora }\end{array}$ & $\begin{array}{l}\text { Senna tora } \\
\text { (L.) Roxb. }\end{array}$ & $\begin{array}{l}\text { Legumino- } \\
\text { sae }\end{array}$ & Herb & Monsoon & $\begin{array}{l}\text { Leaves } \\
\text { and Seeds }\end{array}$ & Food & $\begin{array}{l}\text { 1. Young leaves boiled and } \\
\text { cooked as vegetable } \\
\text { 2. Seeds roasted and } \\
\text { powdered to prepare coffee } \\
\text { powder and then consumed } \\
\text { with boiled water or milk. }\end{array}$ & - \\
\hline
\end{tabular}




\begin{tabular}{|c|c|c|c|c|c|c|c|c|c|}
\hline $\begin{array}{l}\text { Sr. } \\
\text { No }\end{array}$ & $\begin{array}{l}\text { Local } \\
\text { Name of } \\
\text { Species }\end{array}$ & $\begin{array}{l}\text { Scientific } \\
\text { Name }\end{array}$ & Family & Habit & $\begin{array}{l}\text { Season } \\
\text { of availa- } \\
\text { bility }\end{array}$ & $\begin{array}{l}\text { Plant } \\
\text { part } \\
\text { used }\end{array}$ & $\begin{array}{l}\text { Signifi- } \\
\text { cance }\end{array}$ & $\begin{array}{l}\text { Recipes } \\
\text { (If any) }\end{array}$ & $\begin{array}{l}\text { Medicinal } \\
\text { information }\end{array}$ \\
\hline 148. & $\begin{array}{l}\text { Tehra/ } \\
\text { Terda }\end{array}$ & $\begin{array}{l}\text { Impatiens } \\
\text { balsamina L. }\end{array}$ & $\begin{array}{l}\text { Balsamina- } \\
\text { ceae }\end{array}$ & Herb & Monsoon & $\begin{array}{l}\text { Young } \\
\text { leaves }\end{array}$ & Food & $\begin{array}{l}\text { Young leaves used as } \\
\text { vegetable }\end{array}$ & - \\
\hline 149 . & $\begin{array}{l}\text { Tembhru- } \\
\text { ni/ Tem- } \\
\text { bhurni/ } \\
\text { Tembrun }\end{array}$ & $\begin{array}{l}\text { Diospyros } \\
\text { melanoxylon } \\
\text { Roxb. }\end{array}$ & Ebenaceae & Tree & Summer & Fruits & Food & Fruits are consumed directly. & - \\
\hline 150. & $\begin{array}{l}\text { Tetava/ } \\
\text { Tetavi/ } \\
\text { Tetu }\end{array}$ & $\begin{array}{l}\text { Oroxylum } \\
\text { indicum }(\mathrm{L} .) \\
\text { Kurz }\end{array}$ & $\begin{array}{l}\text { Bignonia- } \\
\text { ceae }\end{array}$ & Tree & $\begin{array}{l}\text { Early } \\
\text { Summer }\end{array}$ & $\begin{array}{l}\text { Young } \\
\text { pods and } \\
\text { Bark }\end{array}$ & Food & $\begin{array}{l}\text { Young pods are boiled and } \\
\text { cooked to make vegetable. }\end{array}$ & $\begin{array}{l}\text { Bark decoction is } \\
\text { effective in } \\
\text { urinary infection }\end{array}$ \\
\hline 151. & Tivas & $\begin{array}{l}\text { Desmodium } \\
\text { oojeinense } \\
\text { (Roxb.) } \\
\text { H.Ohashi }\end{array}$ & $\begin{array}{l}\text { Legumino- } \\
\text { sae }\end{array}$ & Tree & $\begin{array}{l}\text { All } \\
\text { season }\end{array}$ & $\begin{array}{l}\text { Flowers } \\
\text { and Bark }\end{array}$ & Food & $\begin{array}{l}\text { Flowers are boiled and } \\
\text { cooked as vegetable. }\end{array}$ & $\begin{array}{l}\text { Bark decoction } \\
\text { helps in balanc- } \\
\text { ing the blood } \\
\text { pressure level }\end{array}$ \\
\hline 152. & $\begin{array}{l}\text { Tondali/ } \\
\text { Tondval }\end{array}$ & $\begin{array}{l}\text { Coccinia } \\
\text { grandis(L.) } \\
\text { Voigt }\end{array}$ & $\begin{array}{l}\text { Cucurbita- } \\
\text { ceae }\end{array}$ & Climber & Monsoon & Fruits & $\begin{array}{l}\text { Food and } \\
\text { Medicinal }\end{array}$ & $\begin{array}{l}\text { Fruits boiled and cooked } \\
\text { as vegetable. }\end{array}$ & $\begin{array}{l}\text { Milky latex is } \\
\text { used for mouth } \\
\text { ulcer. }\end{array}$ \\
\hline 153. & Toran & $\begin{array}{l}\text { Ziziphus } \\
\text { rugosa Lam. }\end{array}$ & $\begin{array}{l}\text { Rhamna- } \\
\text { ceae }\end{array}$ & Climber & Summer & Fruits & Food & Fruits are consumed directly. & - \\
\hline 154. & $\begin{array}{l}\text { Tulas/ } \\
\text { Tulasi/ } \\
\text { Krushna- } \\
\text { tulas }\end{array}$ & $\begin{array}{l}\text { Ocimum } \\
\text { tenuiflorum } \\
\text { L. }\end{array}$ & Lamiaceae & Herb & $\begin{array}{l}\text { All } \\
\text { season }\end{array}$ & Leaves & $\begin{array}{l}\text { Food and } \\
\text { Medicinal }\end{array}$ & $\begin{array}{l}\text { Tulsi leaves are added } \\
\text { while making tea to add } \\
\text { flavor }\end{array}$ & $\begin{array}{l}\text { Leaf extract is } \\
\text { consumed during } \\
\text { headaches and } \\
\text { coughs. }\end{array}$ \\
\hline 155. & Udid & $\begin{array}{l}\text { Vigna } \\
\text { radiata(L.) } \\
\text { R.Wilczek }\end{array}$ & $\begin{array}{l}\text { Legumino- } \\
\text { sae }\end{array}$ & Climber & Monsoon & Seeds & Food & $\begin{array}{l}\text { Seeds are boiled with local } \\
\text { masalas to prepare curry. }\end{array}$ & - \\
\hline
\end{tabular}




\begin{tabular}{|c|c|c|c|c|c|c|c|c|c|}
\hline $\begin{array}{l}\text { Sr. } \\
\text { No }\end{array}$ & $\begin{array}{l}\text { Local } \\
\text { Name of } \\
\text { Species }\end{array}$ & $\begin{array}{l}\text { Scientific } \\
\text { Name }\end{array}$ & Family & Habit & $\begin{array}{l}\text { Season } \\
\text { of availa- } \\
\text { bility }\end{array}$ & $\begin{array}{l}\text { Plant } \\
\text { part } \\
\text { used }\end{array}$ & $\begin{array}{l}\text { Signifi- } \\
\text { cance }\end{array}$ & $\begin{array}{l}\text { Recipes } \\
\text { (If any) }\end{array}$ & $\begin{array}{l}\text { Medicinal } \\
\text { information }\end{array}$ \\
\hline 156. & $\begin{array}{l}\text { Ulashi/ } \\
\text { Udasha/ } \\
\text { Ulushi }\end{array}$ & $\begin{array}{l}\text { Dioscorea } \\
\text { pentaphylla } \\
\text { L. }\end{array}$ & $\begin{array}{l}\text { Dioscorea- } \\
\text { ceae }\end{array}$ & Climber & Monsoon & $\begin{array}{l}\text { Tender } \\
\text { shoots, } \\
\text { leaves and } \\
\text { tuber }\end{array}$ & $\begin{array}{l}\text { Food and } \\
\text { Medicinal }\end{array}$ & $\begin{array}{l}\text { 1. Tubers are boiled and } \\
\text { consumed as vegetable. } \\
\text { 2. Tender shoots are steamed } \\
\text { and stir fried with onion and } \\
\text { local masalas as vegetable. }\end{array}$ & $\begin{array}{l}\text { Leaf extract is } \\
\text { given as paste } \\
\text { during iron } \\
\text { deficiency. }\end{array}$ \\
\hline 157. & $\begin{array}{l}\text { Umbar/ } \\
\text { Umber/ } \\
\text { Athi }\end{array}$ & $\begin{array}{l}\text { Ficus } \\
\text { racemosa } \mathrm{L} .\end{array}$ & Moraceae & Tree & $\begin{array}{l}\text { All } \\
\text { season }\end{array}$ & Fruits & Food & $\begin{array}{l}\text { Raw fruits are used to make } \\
\text { vegetable while ripe fruits } \\
\text { are consumed directly. }\end{array}$ & - \\
\hline 158. & Usiver & $\begin{array}{l}\text { Cayratia } \\
\text { japonica } \\
\text { (Thunb.) } \\
\text { Gagnep. }\end{array}$ & Vitaceae & Climber & Monsoon & Flowers & Food & $\begin{array}{l}\text { Flowers are cooked as } \\
\text { vegetables. }\end{array}$ & - \\
\hline 159. & Vad & $\begin{array}{l}\text { Ficus } \\
\text { benghalensis } \\
\text { L. }\end{array}$ & Moraceae & Tree & Monsoon & Fruits & Medicinal & - & $\begin{array}{l}\text { Fruits are } \\
\text { consumed during } \\
\text { stomach pain. }\end{array}$ \\
\hline 160. & $\begin{array}{l}\text { Vaghot/ } \\
\text { Vaghat/ } \\
\text { Vaghoti/ } \\
\text { Ekota/ } \\
\text { Kaposhi }\end{array}$ & $\begin{array}{l}\text { Capparis } \\
\text { zeylanica } \mathrm{L} .\end{array}$ & $\begin{array}{l}\text { Cappara- } \\
\text { ceae }\end{array}$ & Climber & Winter & Fruits & Food & $\begin{array}{l}\text { Raw fruits are cooked and } \\
\text { consumed as vegetable. }\end{array}$ & - \\
\hline 161. & $\begin{array}{l}\text { Vela } \\
\text { karanj }\end{array}$ & $\begin{array}{l}\text { Derris } \\
\text { scandens } \\
\text { (Roxb.) } \\
\text { Benth. }\end{array}$ & $\begin{array}{l}\text { Legumino- } \\
\text { sae }\end{array}$ & Climber & Monsoon & Tuber & Medicinal & - & $\begin{array}{l}\text { Tuber decoction } \\
\text { is given to } \\
\text { lactating } \\
\text { mothers. }\end{array}$ \\
\hline 162. & Zendu & $\begin{array}{l}\text { Tagetes } \\
\text { erecta } \mathrm{L} .\end{array}$ & Compositae & Herb & $\begin{array}{l}\text { All } \\
\text { season }\end{array}$ & Leaves & Medicinal & - & $\begin{array}{l}\text { Decoction of } \\
\text { leaves is used to } \\
\text { cure eye infection }\end{array}$ \\
\hline
\end{tabular}

